\title{
Quasi-simultaneous multi-frequency observations of inverted-spectrum GPS candidate sources $\star, \star \star$
}

\author{
B. Vollmer ${ }^{1}$, T. P. Krichbaum ${ }^{2}$, E. Angelakis ${ }^{2}$, and Y. Y. Kovalev ${ }^{2,3}$
}

1 CDS, Observatoire astronomique de Strasbourg, 11 rue de l'université, 67000 Strasbourg, France e-mail: bvollmer@astro.u-strasbg.fr

2 Max-Planck-Insitut für Radioastronomie, Auf dem Hügel 69, 53121 Bonn, Germany

3 Astro Space Center of Lebedev Physical Institute, Profsoyuznaya 84/32, 117997 Moscow, Russia

Received 16 October 2007 / Accepted 3 June 2008

\section{ABSTRACT}

\begin{abstract}
Context. Gigahertz-Peaked Spectrum (GPS) sources are likely the precursors of local radio galaxies. Existing GPS source samples are small $(<200)$

Aims. We aim to extend the available sample of the Gigahertz-Peaked Spectrum (GPS) and High Frequency Peaker (HFP) sources in order to study their nature with greater detail and higher statistical significance.

Methods. A sample of 214 radio sources, which were extracted from the SPECFIND catalog and show an inverted radio spectrum, were observed quasi-simultaneously at $4.85,10.45$, and $32 \mathrm{GHz}$ with the 100-m Effelsberg radio telescope. Using the VLBA calibrator survey (VCS) we have investigated the parsec-scale morphology of the sources.

Results. About $45 \%$ of the sources in our sample are classified as GPS or HFP candidates. We add 65 new GPS/HFP candidates to existing samples. We confirm the expected tendency that HFP are more compact on a milliarcsecond scale than the "classical" GPS sources, which peak at lower frequencies.

Conclusions. The data mining of the SPECFIND database represents a promising tool for the discovery of new GPS/HFP sources.
\end{abstract}

Key words. radio continuum: galaxies

\section{Introduction}

GHz-Peaked Spectrum (GPS) sources are powerful $\left(\log P_{1.4 \mathrm{GHz}}>25 \mathrm{~W} \mathrm{~Hz}^{-1}\right)$ and compact $(<1 \mathrm{kpc})$ extragalactic radio sources, which show a convex radio spectrum peaking between $500 \mathrm{MHz}$ and $10 \mathrm{GHz}$ in the observer's frame (for a review see O'Dea 1998). The physical mechanism responsible for the turnover of the spectrum is still unclear with two competing models proposed: the synchrotron self-absorption caused by dense plasma within the source or the free-free absorption caused by a screen external to the source.

High Frquency Peakers (HFP) are radio sources defined via their convex spectrum peaking at frequencies above $5 \mathrm{GHz}$ (Dallacasa et al. 2000). From the anti-correlation found between the turnover frequency and size (O'Dea \& Baum 1997) HFPs are expected to be smaller and therefore younger radio sources than GPS sources.

GPS sources are associated with either quasars or galaxies. Despite the similar shape of their radio spectrum, these two classes of GPS sources are often considered to be different. Torniainen et al. (2005), who studied the long term variability of 35 inverted-spectrum sources, concluded that genuine quasartype GPS sources are rare. They found a large number of highly variable blazar sources that can have a convex spectrum

\footnotetext{
* Tables 5 and 6 are only available in electronic form at the CDS via anonymous ftp to cdsarc.u-strasbg.fr (130.79.128.5) or via http://cdsweb.u-strasbg.fr/cgi-bin/qcat?J/A+A/489/49

$\star \star$ Full Fig. 4 is only available in electronic form at

http://www . aanda.org
}

peaking at high frequencies (up to $\sim 100 \mathrm{GHz}$ ) during flaring events occurring in the radio jets.

The nature of GPS sources is still under debate. Two possible scenarios have been put forth: (i) the "frustration" scenario, according to which the small size and the inverted spectrum are caused by a dense environment that prohibits the source from growing larger (e.g., Gopal-Krishna \& Wiita 1991); (ii) the "youth" scenario, suggesting that the GPS sources represent the young precursors of compact steep spectrum (CSS) sources and extended radio sources (e.g., Mutel \& Phillips 1988; Fanti et al. 1990, 1995).

There is now a wide consensus that, at least the symmetric GPS sources, represent the early evolutionary stage of the extended radio source population. At this stage the radio emitting region grows and expands within the interstellar medium before plunging into the intergalactic medium to form an FR II radio source (Fanti et al. 1995; Readhead et al. 1996; Begelman 1996; Snellen et al. 2000a).

The detection of kpc-scale emission associated with a few GPS sources seems to be inconsistent with a recent origin of the radio activity. Such an extended emission is interpreted as a sign of a past nuclear activity. In this case the GPS source, i.e. the galactic nucleus, is at the beginning of a new cycle of activity. Since extended radio emission around GPS sources is a rare phenomenon, Stanghellini et al. (2005) conclude that the time scale between subsequent phases of activity is in general longer than the radiative lifetime of the radio emission from the earlier activity $\left(\sim 10^{8} \mathrm{yr}\right)$.

The currently existing GPS sample is limited $(<200$ objects, see, e.g., the recent compilation by Labiano et al. 2007). In order 
to conduct statistical studies of GPS objects and test whether the number of GPS sources at intermediate redshifts is in accordance with that of local FR II radio galaxies, it is necessary that the sample of available GPS sources is extended. In particular, this could help to test whether GPS sources are the precursors of the local FR II radio galaxies.

In this paper, we present results of a search for new GPS/HFP candidates. Since the identification of a radio source as a genuine young GPS source depends on the detailed knowledge of its spectrum, flux density variability, VLBI structure and source identification, we will use in the following a GPS or HFP source as a synonym for GPS candidate or HFP candidate. In order to measure radio spectra over a range of high frequencies, which are not affected by non-contemporanous measurements, we observed a sample of 214 objects north of declination $-25^{\circ}$ that show an inverted radio spectrum, with quasi-simultaneous flux density measurements at $4.85 \mathrm{GHz}$, $10.45 \mathrm{GHz}$, and $32 \mathrm{GHz}$ using the Effelsberg 100-m telescope of the Max-Planck-Institut für Radioastronomie (MPIfR). In Sect. 2 we review the sample selection procedure implemented on the basis of the SPECFIND database, observations and primary data reduction are described in Sect. 3. We present results of observations in Sect. 4, sources extention and variablility characteristics in Sect. 5, cross-identification and spectral fitting in Sect. 6, milliarsecond-scale compactness and morphology in Sect. 7, comparison with other available GPS/HFP samples in Sect. 8. We summarize our results in Sect. 9.

\section{Sample selection}

SPECFIND (Vollmer et al. 2005a) is a hierarchical algorithm. It classifies a source $j$ as parent, sibling, or child with respect to a given source $i$ using a procedure that has different stages:

Stage 1: cross-identification based on proximity criteria taking into account the resolution and source size;

Stage 2: cross-identification based on flux densities at the same frequency;

Stage 3: cross-identification based on flux density at different frequencies, as expected from the radio spectral index, adopting the convention $S_{v} \propto v^{\alpha}$.

At the end of this procedure a source $i$ and its siblings are considered to be the same source. If source $j$ is identified as a parent, source $i$ might be a resolved sub-source of source $j$. If source $i$ has children, it might be extended, and the children represent its resolved sub-sources. At the end of the cross-identification the self-consistency of the hierarchy and the uniqueness is tested. A radio source cannot be assigned to more than one physical object. The position of each object is taken from the radio source observed with the highest resolution (for the positional accuracy of the included radio surveys see Vollmer et al. 2005b).

The result of the cross-identification depends strongly on the detailed spectrum fitting algorithm (Vollmer et al. 2005a), the initial set of frequency points and their weights. During this procedure, non-fitted frequency points are rejected and removed from the spectrum, since they may belong to a different object. SPECFIND determines the slope and the abscissa for a given source $i$ with all other cross-identified sources. It is required that the final power law fits source $i$ within the errors. Therefore, the result of the fitting algorithm can be different depending on the choice of source $i$. A given object with an associated radio spectrum can thus possess several slopes and abscissa.

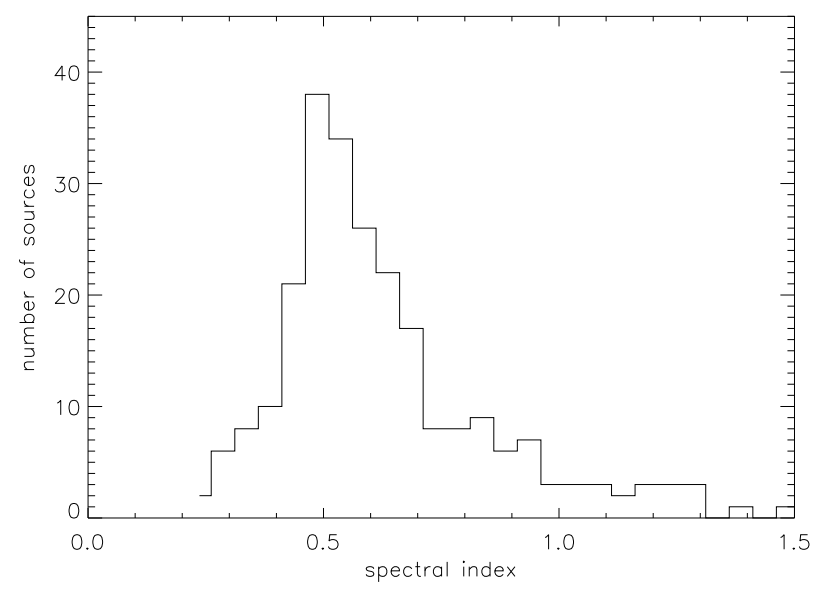

Fig. 1. SPECFIND spectral index distribution of the sources in our sample. The power law spectrum is defined by $S_{v} \propto v^{\alpha}$. All the objects have several spectral indices depending on the weighting of the frequency points. Objects with $\alpha<0.5$ have at least one associated spectral index $\geq 0.5$.

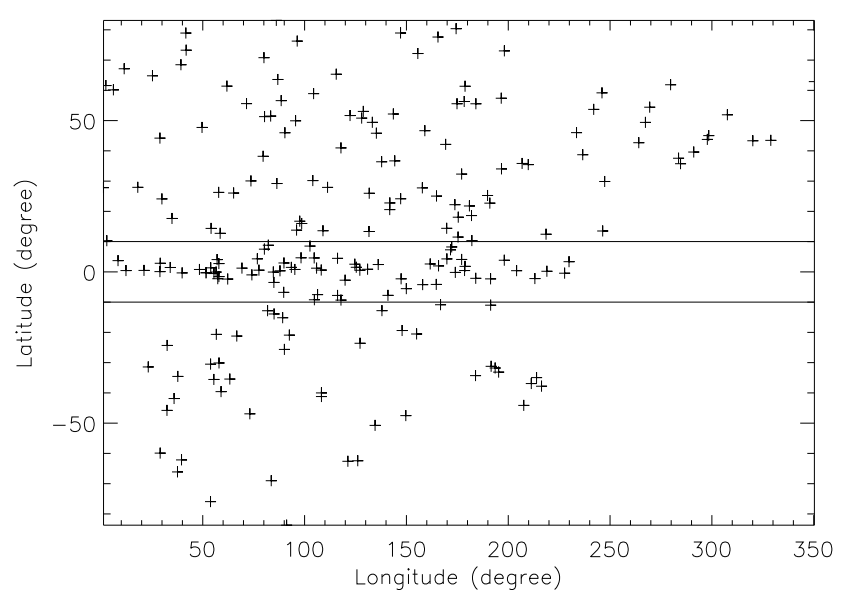

Fig. 2. Spatial distribution of our sample of 214 sources with an inverted radio spectrum in Galactic coordinates. The horizontal lines mark Galactic latitudes of $\pm 10^{\circ}$.

From an extended version of the SPECFIND catalog ${ }^{1}$, which contains the comparison samples described in Table 5 of Vollmer et al. (2005a), we extracted objects with a maximum slope (spectral index, $\alpha$ ) greater than or equal to 0.5 and a declination $\delta>-25^{\circ}$. The frequency range in which the spectral index is determined depends on the actual frequency coverage and sensitivity of the 22 individual radio catalogues used by SPECFIND. To avoid confusion problems for the flux density measurements performed at Effelsberg, we then removed all sources that are extended in the NVSS (larger than the 45" beam size; Condon et al. 1998) and some Galactic sources, which coincide with known HII regions.

This resulted in a list of 214 objects. The majority of the sources has a spectral index of around +0.5 . The distribution of spectral indices of these sources is shown in Fig. 1, and their distribution on the sky is shown in Fig. 2. About one third of them (69) are located within $\pm 10^{\circ}$ from the Galactic plane. Some of them were later identified as infrared sources or planetary nebula. Since our selection criterion is only based on the shape of the radio spectrum, we did not remove these sources. The number of targets is smaller in the southern hemisphere than in the

1 Available at CDS VizieR (Ochsenbein et al. 2000). 
Table 1. Effelsberg observing sessions.

\begin{tabular}{rc}
\hline \hline Date & UT range $(\mathrm{h})$ \\
\hline 23, 24 Sep. 2005 & $11-08$ \\
8, 9 Oct. 2005 & $07-04$ \\
17, 18 Jan. 2006 & $18-02$ \\
24, 25 Jan. 2006 & $16-08$ \\
30, 31 Jan. 2006 & $15-08$ \\
24 Mar. 2006 & $02-10$ \\
\hline
\end{tabular}

northern hemisphere because of the lack of deep surveys in the southern hemisphere (Vollmer et al. 2005a).

\section{Observations and primary data reduction}

The flux density measurements of the 214 target sources were made with the 100-m MPIfR radio telescope in Effelsberg (Germany). The observations took place in 6 observing sessions which are summarized in Table 1.

In each session the target sources and the calibrator sources (see Table 2) were observed with the heterodyne receivers mounted at the secondary focus of the telescope. The measurements were made at $6 \mathrm{~cm}(4.85 \mathrm{GHz}), 2.8 \mathrm{~cm}(10.45 \mathrm{GHz})$ and $9 \mathrm{~mm}(32 \mathrm{GHz})$. The receiver characteristics are summarized in Table 3. Column 1 gives the observing wavelength, Col. 2 the central frequency, Col. 3 the observing bandwidth, Col. 4 the typical system temperature (at zenith), Col. 5 the typical peak value of the elevation-dependent antenna gain, Col. 6 the size of the observing beam (full width at half maximum, $F W H M$ ), Col. 7 the polarization of the receiver feeds, Col. 8 the number of receiver horns used in the data reduction ${ }^{2}$, and Col. 9 the receiver type. The signal from the second receiver horn allowed the off-source signal containing the (time variable) atmospheric signal to be subtracted from the signal on the source (beam switch). This improved the data quality and led to almost flat baselines in each scan.

For each source, the antenna temperature was measured using the method of "cross-scans". Here, the telescope beam is repeatedly moved across the source position, with an equal number of sub-scans in elevation and in the azimuth direction. The number of sub-scans in each cross-scan ranged between 4-12, depending on the flux density of the source and the stability of the telescope pointing. Each source was measured with at least two cross-scans at each of the three observing bands. With a typical duration of 25-30 s for each sub-scan and scan lengths of 600", $220^{\prime \prime}$, and $110^{\prime \prime}$ at $\lambda=6 \mathrm{~cm}, 2.8 \mathrm{~cm}$, and $9 \mathrm{~mm}$, respectively, each scan lasted between 3-5 min. This allowed us to obtain for each source "quasi-simultaneous" flux density measurements at the 3 observing bands within a 15-20 min time interval.

After the observations, the data were reduced in the standard manner following the procedures described in Kraus et al. (2003). To derive the flux densities of the observed point sources, Gaussian profiles were fitted to each sub-scan in each driving direction. For fainter sources, the sub-scans were averaged in each driving direction before the Gaussian fitting was done. After correcting for small residual pointing offsets, the amplitudes of the individual sub-scans were combined by averaging them. For each scan and source this resulted in the uncalibrated antenna temperature as determined by the peak value, $S^{\text {peak }}$, of the Gaussian fit. For each scan, the half-power beam

2 The $6-\mathrm{cm}$ receiver has 2 feeds, the $2.8-\mathrm{cm}$ one has 4 and the $9 \mathrm{~mm}$ receiver has 6 feeds.
Table 2. The primary and the secondary calibrators along with and their flux densities at the observing frequencies.

\begin{tabular}{lcccl}
\hline \hline Source & $\begin{array}{c}S_{6 \mathrm{~cm}} \\
{[\mathrm{Jy}]}\end{array}$ & $\begin{array}{c}S_{2.8 \mathrm{~cm}} \\
{[\mathrm{Jy}]}\end{array}$ & $\begin{array}{c}S_{9 \mathrm{~mm}} \\
{[\mathrm{Jy}]}\end{array}$ & Comment \\
\hline 3C 48 & 5.48 & 2.60 & 0.80 & Primary Calibrator \\
3C 138 & 3.79 & 2.16 & 0.92 & Secondary Calibrator \\
3C 147 & 7.90 & 3.82 & 1.18 & Secondary Calibrator \\
3C 161 & 6.62 & 3.06 & 0.83 & Secondary Calibrator \\
3C 286 & 7.48 & 4.45 & 1.82 & Primary Calibrator \\
3C 295 & 6.56 & 2.62 & 0.55 & Primary Calibrator \\
NGC 7027 & 5.48 & 5.92 & 5.49 & Primary Calibrator \\
\hline
\end{tabular}

width, $H P B W^{\text {obs }}$ also, was measured. In the next step we corrected the measurements for the elevation-dependent antenna gain and for systematic time-dependent effects, using standard gain-elevation curves and the known flux densities of the primary and secondary calibrators, which where observed regularly at 3-6 h intervals throughout each of the observing sessions. Opacity corrections were applied to the $9 \mathrm{~mm}$ data. The conversion from antenna temperature (measured in $\mathrm{K}$ ) to flux density (measured in Jy) was done using the flux density scale of Ott et al. (1994), refined by more recent measurements (Kraus, priv. comm.). Table 2 summarizes the flux density scale used throughout this paper.

\section{Results}

The observed radio flux densities at 4.85, 10.45, and $32 \mathrm{GHz}$ are listed in Table 5 along with the spectral indices between 4.85-10.45 GHz and $10.45-32 \mathrm{GHz}$. The last column of the table lists the observing frequency in $\mathrm{GHz}$, at which the FWHM of the Gaussian fitted to the telescope response was significantly larger than that to the point source (see Table 3). This is indicative of extended source structure or confusion. The sources marked in this way should be regarded either as partially resolved or as being confused within the Effelsberg observing beam. We detected all 214 sources at $4.85 \mathrm{GHz}, 209$ (98\%) sources at $10.45 \mathrm{GHz}$, and $181(85 \%)$ sources at $32 \mathrm{GHz}$. For the non-detected sources we assign a dash ("-") in the corresponding column of Table 5. Depending on the on-source integration time, receiver properties and weather conditions the estimated detection limits are $3 \sigma=1-5 \mathrm{mJy}$ at $4.85 \mathrm{GHz}, 3-10 \mathrm{mJy}$ at $10.45 \mathrm{GHz}$ and $10-30 \mathrm{mJy}$ at $32 \mathrm{GHz}$, with the higher numbers of each range reflecting non-optimal weather conditions. The provided errors of the flux density measurements are $1 \sigma$ errors and result from formal error propagation through the following steps: Gaussian fitting, gain and time correction, K/Jy conversion. The error of the absolute flux density scale (Baars et al. 1977; Ott et al. 1994) is not included in the provided errors. The error of the spectral indices follows from the Gaussian error propagation of the flux density errors.

The derived distributions of the spectral indices are shown in Fig. 3. The number of sources with a negative spectral index between 4.85 and $10.45 \mathrm{GHz}$ is 142 , that of sources with a negative spectral index between $10.45 \mathrm{GHz}$ and $32 \mathrm{GHz}$ is 139 . The number of sources with a positive spectral index between 4.85 and $10.45 \mathrm{GHz}$ is $67(31 \%)$, whereas 41 sources show a positive spectral index between $10.45 \mathrm{GHz}$ and $32 \mathrm{GHz}$. Thus, $\sim 20 \%$ of the sources of our sample show an inverted spectrum up to $32 \mathrm{GHz}$. Out of 214 sources 63 (29\%) are resolved by the Effelsberg beam at one or more observing frequencies. Out of the 63 resolved objects 30 are within $10^{\circ}$ from the Galactic 
Table 3. The characteristics of the receivers used.

\begin{tabular}{ccccccccc}
\hline \hline $\begin{array}{c}\lambda \\
{[\mathrm{cm}]}\end{array}$ & $\begin{array}{c}\text { Center } v \\
{[\mathrm{GHz}]}\end{array}$ & $\begin{array}{c}\text { Bandwidth } \\
{[\mathrm{MHz}]}\end{array}$ & $\begin{array}{c}T_{\text {sys }} \\
{[\mathrm{K}]}\end{array}$ & $\begin{array}{c}\text { Gain } \\
{[\mathrm{K} / \mathrm{Jy}]}\end{array}$ & $\begin{array}{c}F W H M \\
{\left[{ }^{\prime \prime}\right]}\end{array}$ & Polarization & Horns & Comment \\
\hline 6 & 4.85 & 500 & 27 & 1.5 & 146 & dual circ. & 2 & Software Beam switch \\
2.8 & 10.45 & 300 & 50 & 1.3 & 67 & dual circ. & 2 & Software Beam switch \\
0.9 & 32.00 & 2000 & 77 & 0.5 & 26 & LCP & 2 & Correlation Receiver \\
\hline
\end{tabular}
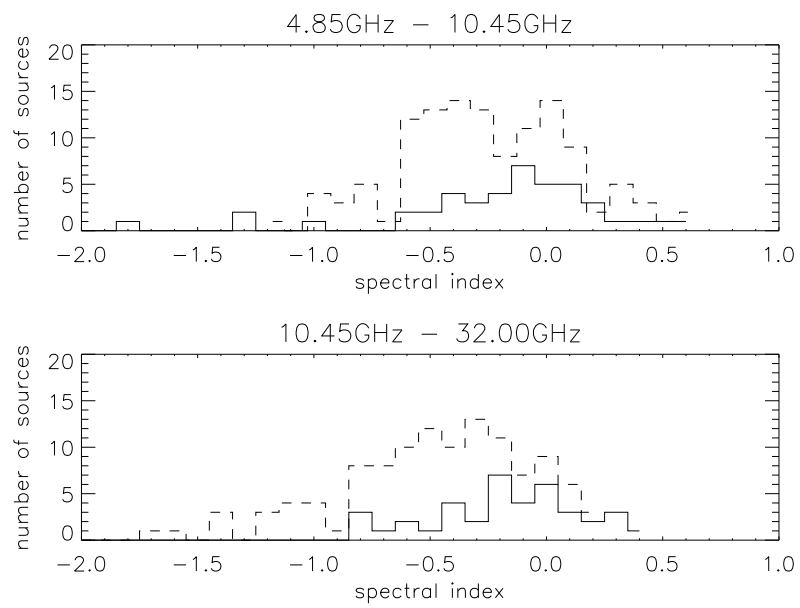

Fig. 3. The spectral index distribution for variable (solid line) and nonvariable (dashed line) sources. The spectral indices have been calculated from flux densities at $4.85 \mathrm{GHz}$ and $10.45 \mathrm{GHz}$ (upper panel) and at $10.45 \mathrm{GHz}$ and $32.00 \mathrm{GHz}$ (lower panel).

plane. Identification statistics about the object types are given in Sect. 8.

\section{Extended sources and variability}

The source spectra extracted with SPECFIND from the database result from non-simultaneous multi-epoch flux density measurements with different telescopes. The observations described here, however, have been obtained with the 100-m Effelsberg telescope, providing for each source quasi-simultaneous measurements within 15-20 min at 3 different frequencies. One expects that radio sources with flat or inverted spectra show a higher degree of variability than those with steeper spectra. In order to test this idea, we compared the Effelsberg $4.85 \mathrm{GHz}$ $(6 \mathrm{~cm})$ flux density to a corresponding $4.85 \mathrm{GHz}$ flux density in SPECFIND whenever that is available (121 sources of our sample). The SPECFIND used $4.85 \mathrm{GHz}$ flux densities obtained with angular resolutions of $3.5^{\prime}$ and 2.8' (Gregory et al. 1996; Gregory \& Condon 1991; Becker et al. 1991; Wright et al. 1994, 1996; Griffith et al. 1994, 1995; Bennett et al. 1986; Langston et al. 1990; Griffith et al. 1990, 1991), compared to the Effelsberg $100-\mathrm{m}$ telescope resolution of $2.4^{\prime}$. Therefore, extended or confused sources observed with these different beam sizes can also lead to differences between the Effelsberg and the SPECFIND fluxes.

Since the SPECFIND catalog may contain more than one flux density at $4.85 \mathrm{GHz}$, we base our variability/confusion criterion on the maximum of the absolute value of the differences between the SPECFIND and the Effelsberg 4.85 GHz flux densities $\Delta S_{4.85 \mathrm{GHz}}=S_{4.85 \mathrm{GHz}}^{\mathrm{Eff}}-S_{4.85 \mathrm{GHz}}^{\text {SPECFIND }}$ :

$\max \left(\left|\Delta S_{4.85 \mathrm{GHz}}\right|\right) / S_{4.85 \mathrm{GHz}}^{\mathrm{Eff}} \geq 0.5$,

where $S_{4.85 \mathrm{GHz}}^{\mathrm{Eff}}$ is the Effelsberg and $S_{4.85 \mathrm{GHz}}^{\text {SPECFND }}$ is the SPECFIND flux density at $4.85 \mathrm{GHz}$. In addition, we require no overlap between the SPECFIND and Effelsberg $4.85 \mathrm{GHz}$ points within the errors. After applying this criterion we find that 59 out of 197 sources are suspected to be variable or confused. This represents $30 \%$ of the restricted sample (197 sources having a SPECFIND frequency point at $4.85 \mathrm{GHz}$ ) and $28 \%$ of the whole sample (214 sources). Based on these numbers we expect about $30 \%$ of the sources in our sample to be variable at centimeter wavelengths. Out of the identified 59 variable sources, 10 are quasars, 4 are BL Lacertae object, 3 are galaxies, 3 are X-ray sources, 4 are infrared sources, and 1 is a planetary nebula (PN). As expected, the variable sources tend to be either BL Lacs or quasars (see also Torniainen et al. 2005).

The information about the source extension and variability can be found in Col. 7 of Table 6 . There, the apparently variable/confused sources based on the $4.85 \mathrm{GHz}$ flux densities are marked with the symbol "V". The letter "E" indicates sources whose extensions are larger than the beam of the Effelsberg telescope at at least one frequency. We find 30 variable sources which are extended or confused in at least one of our Effelsberg observations (designated as "EV"). Figure 3 shows the spectral index distribution for variable and non-variable sources. Variable sources have in general a flatter spectral index between 4.85-10.45 GHz and 10.45-32 GHz. We did not test for variability at $10.45 \mathrm{GHz}$ and $32 \mathrm{GHz}$, because there are not many measurements available in the database at these frequencies to compare.

\section{Cross-identification and GPS spectrum fitting}

Figure 4 shows the combined radio spectra from our Effelsberg observations (diamonds) and the SPECFIND catalog (plus signs with error bars) along with the SPECFIND power law spectrum (dashed line). We cross-identified our sources with the CDS SIMBAD database (Wenger et al. 2000). The obtained object types are indicated at the top of each panel and in Table 6. We have cross-identified 108 objects: 45 quasars, 7 Seyfert or LINER galaxies, 8 BL Lacs, 15 galaxies, $8 \mathrm{X}$-ray sources, 8 infrared sources, 8 planetary nebulae, 2 stars, and 2 HII regions. Additionally, we added 5 quasar identifications from Véron \& Véron (2006). As one can see in this list, there are 12 confirmed Galactic sources. As expected, young planetary nebulae and compact HII regions can also show an inverted radio spectrum up to the $\mathrm{GHz}$ regime. In addition, the infrared sources located at $\pm 10^{\circ}$ from the Galactic plane, which are all extended, are most probably also of Galactic nature. We end up with 80 confirmed extragalactic sources with $60 \%$ of them being quasars (from SIMBAD and Véron \& Véron 2006).

Following Snellen et al. (1998) the combined radio spectra were fitted by the function:

$S(v)=\frac{S_{\max }}{1-\mathrm{e}^{-1}} \times\left(\frac{v}{v_{\max }}\right)^{k} \times\left(1-\mathrm{e}^{-\left(\frac{v}{v_{\max }}\right)^{l-k}}\right)$,

where $k$ is the optically thick spectral index, $l$ the optically thin spectral index, and $S_{\max }$ and $v_{\max }$ respectively the peak flux density and peak frequency. The optically thick spectral index $k$ 

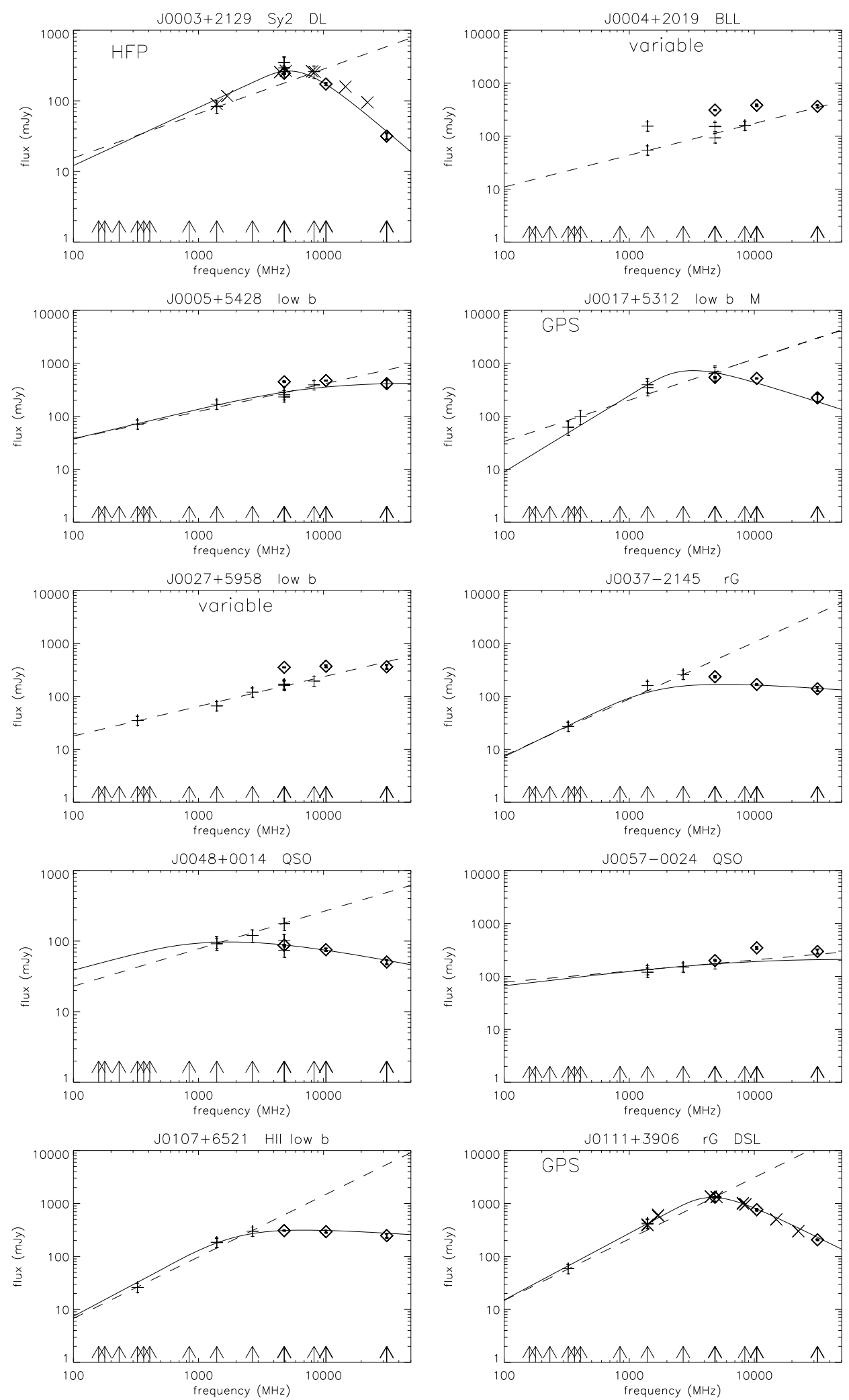

Fig. 4. Radio spectra. SPECFIND data are plotted as pluses with associated error bars, the Effelsberg flux densities as diamonds, and the flux densities obtained by Dallacasa et al. (2000) as crosses. Dashed line: power law fitted by SPECFIND; solid line: fitted spectrum (Eq. (2)). GPS: GHz-Peaked Spectrum; HFP: High Frequency Peaker. On top of each panel the source name, object type (if available), and membership of an existing sample (Table 4) are given. A source located $\pm 10^{\circ}$ from the Galactic plane is labeled with "low b". The arrows on the frequency axis indicate the frequencies of the radio catalogs included in SPECFIND (Vollmer et al. 2005a). The full figure is available in electronic form.

was set to the SPECFIND spectral index (slope of the dashed line in Fig. 4) or to the spectral index between the two points with the lowest frequencies, if this allowed a better fit. For the optically thin spectral index, we used the spectral index 




Fig. 5. Distribution of peak flux densities of GPS sources from Table 6.

between the two points at the highest frequencies available. The peak flux density and peak frequency were then determined with a least square fit method. We also determine the $F W H M$ and $\Delta=\log (F W H M(\mathrm{MHz}))$ of the fitted function (Eq. (2)). A radio source is defined as a GPS if $0<\Delta \leq 2.5$ and $v_{\max } \leq 5 \mathrm{GHz}$. Following Dallacasa et al. (2000) sources with $0<\Delta \leq 2.5$ and $v_{\max }>5 \mathrm{GHz}$ or sources with $\alpha>0.5$ between 1.4 and $5 \mathrm{GHz}$ and $\alpha>0$ between 10 and $32 \mathrm{GHz}$ are classified as High Frequency Peakers (HFP). With these definitions we find 38 GPS and 53 HFP sources. The fitted peak or turnover frequencies can be found in Col. 8 of Table 6 . The distribution of peak flux densities is shown in Fig. 5. Most of our sources have a peak flux density around 0.2 Jy. Our GPS/HFP candidate source sample (Table 6) comprises 18 extended/confused sources and 4 variable sources (Eq. (1)). These objects are likely blazars rather than genuine young GPS/HFP sources.

\section{Milliarcsecond-scale properties}

To investigate parsec-scale characteristics of the sample, we have measured the VLBI compactness at 2.3 and $8.6 \mathrm{GHz}$ as described in Kovalev et al. (2005) using the VLBA ${ }^{3}$ Calibrator Survey (VCS, Beasley et al. 2002; Fomalont et al. 2003; Petrov et al. 2005, 2006, 2008; Kovalev et al. 2007). The compactness is defined as the ratio of the correlated flux density measured at long interferometer spacings ( $u v$-spacings) and the integrated flux density from a VLBI image. Typical errors of the compactness determination are about 0.05 . The error can rise to 0.1 for the sources with integrated flux density about or less than $0.1 \mathrm{Jy}$.

Our sample contains 108 sources from the VLBA Calibrator Survey (Table 6 and Fig. 6). For further analysis it is convenient to discriminate between almost point-like and partially resolved sources. We define a source as (almost) point-like if its compactness is larger than 0.7 . While more than half of the sources are compact at $2.3 \mathrm{GHz}$, the distribution of the compactness at 8.6 GHz is approximately flat (Fig. 6).

The majority of HFP candidate sources (21 out of 35$)$ appear point-like at $2.3 \mathrm{GHz}$ whereas 14 out of $21 \mathrm{GPS}$ candidate

\footnotetext{
3 Very Long Baseline Array of the National Radio Astronomy Observatory.
}
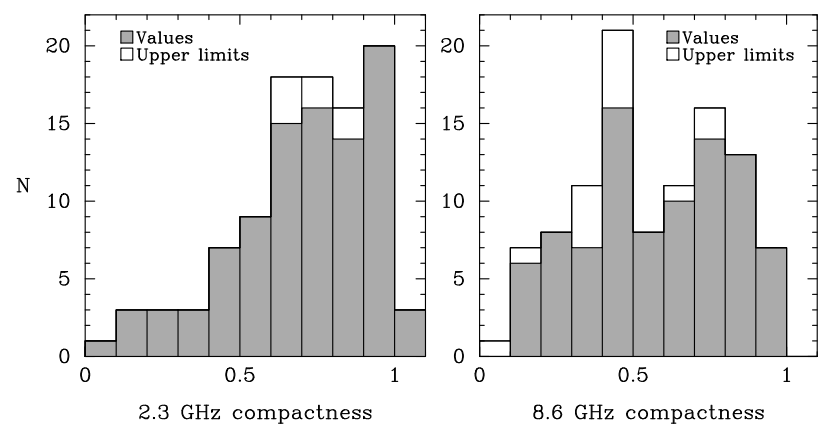

Fig. 6. VLBI compactness ratios at $2.3 \mathrm{GHz}$ (left panel) and $8.6 \mathrm{GHz}$ (right panel).

sources are extended at this frequency. Genuine GPS/HFP candidate sources are expected to be resolved at both frequencies with compactness values decreasing with increasing frequency. We find that 24 out of 53 GPS/HFP candidate sources with available compactness information do show this behavior. Indeed the median compactness values are 0.55 (GPS) and 0.75 (HFP) at $2.3 \mathrm{GHz}$, while at $8.6 \mathrm{GHz}$ they decrease to 0.37 (GPS) and 0.44 (HFP).

In a second step, we classified the sources based on their morphology in the two-frequency VLBI images. This classification is summarized in Col. 13 of Table 6: "nc" - naked core candidate; "cj" - core jet candidate; "cso" - compact symmetric object candidate. A source is classified here as a cso if distinct components of its dual-frequency VLBA structure have 2.3-8.6 GHz spectral index values that differ by less than 0.5 . It is important to note that, on the basis of the two-frequency VLBI data alone, one cannot definitely decide whether an object is a classical compact symmetric one. Rather a multi-band VLBI study is needed in order to reach a definite conclusion (e.g., Peck \& Taylor 2000; Snellen et al. 2000b; Xiang et al. 2002, 2005, 2006; Orienti et al. 2006). A “deeper" VLBI experiment could also detect a weak jet in many sources classified here as naked cores. In summary, we identify 65 previously unknown GPS/HFP candidate sources out of which 32 are listed also in the VLBA Calibrator survey catalog.

In Fig. 7 we show the distribution of the turnover frequencies for the different VLBI morphology classes. Most of the sources are point-like (naked cores), about half of this number have additional jets detected and 9 sources are classified as compact symmetric object candidates. The CSO candidates that are extended at the milliarcsecond-scale tend to have lower values of the turnover frequency in comparison to the properties of the full sample.

\section{Comparison with other GPS/HFP samples}

In order to investigate if our objects already have been identified as GPS/HFP sources, we cross-identified our sample with existing GPS/HFP samples of Snellen et al. (1998), Stanghellini et al. (1998), Marecki et al. (1999), Dallacasa et al. (2000), Fanti et al. (2001) and Labiano et al. (2007). Table 4 shows the results of this cross-identification. The sample designations can also be found in Col. 6 of Table 6 and on top of each plot in Fig. 4. We find 32 previously known GPS/HFP sources in our sample, out of which 26 are classified as GPS/HFP candidates by us. Dallacasa et al. (2000) made quasi-simultaneous multi-frequency observations of HFP sources at the VLA. We have plotted their flux densities as crosses on the spectra of Fig. 4. For the majority of 




Fig. 7. The distribution of the turnover frequencies for the different VLBI morphology classes.

Table 4. Cross-identification with existing GPS/HFP samples.

\begin{tabular}{lcc}
\hline \hline Article & Designation & Number of objects \\
\hline Snellen et al. (1998) & $\mathrm{B}$ & 3 \\
Stanghellini et al. (1998) & $\mathrm{S}$ & 7 \\
Fanti et al. (2001) & $\mathrm{F}$ & 0 \\
Dallacasa et al. (2000) & $\mathrm{D}$ & 15 \\
Marecki et al. (1999) & $\mathrm{M}$ & 9 \\
Labiano et al. (2007) & $\mathrm{L}$ & 14 \\
\hline
\end{tabular}

the sources there is a good agreement with the Effelsberg and SPECFIND flux densities.

\section{Summary}

A sample of 214 radio sources with inverted spectra and declination $\delta>-25^{\circ}$ was extracted from the SPECFIND catalog (Vollmer et al. 2005a). This catalog contains crossidentifications of radio sources from surveys at different frequencies and combines them into one single radio spectrum per object. To obtain quasi-simultaneous radio spectra, we observed those sources with the MPIfR Effelsberg 100-m radio telescope at $4.85,10.45$, and $32 \mathrm{GHz}$. All the observed sources were detected at $4.85 \mathrm{GHz}$, while 209 and 180 sources were detected at $10.45 \mathrm{GHz}$ and $32 \mathrm{GHz}$, respectively. We expect the fraction of variable or confused sources in our complete sample to be of $\sim 30 \%$. On the basis of the performed analysis of continuum radio spectra, we have identified 38 GPS and 53 HFP candidates out of which 65 were previously unknown. An inspection of VCS data shows that 24 out of 53 GPS/HFP sources with available VCS data are resolved at 2.3 and $8.6 \mathrm{GHz}$. We have confirmed the expected tendency for HFP objects to be highly compact and the GPS ones to show a significantly lower level of compactness at the milliarcsecond scale. This independently supports the robustness of our source classifications presented here.

We have a success rate of $\sim 45 \%$ for finding GPS/HFP candidates from a selected SPECFIND catalog sample. Once the SPECFIND catalog is upgraded with the inclusion of more radio catalogs, this method comprises a promising way for future identification of new GPS/HFP sources.
Acknowledgements. This work is based on observations with the 100-m telescope of the MPIfR (Max-Planck-Institut für Radioastronomie) at Effelsberg. We would like to thank A. Kraus for his help. Y. Y. Kovalev is a Research Fellow of the Alexander von Humboldt Foundation. We would like to thank John McKean for fruitful discussions. We thank the anonymous referee for thoughtful reading and useful comments which helped to improve the manuscript.

\section{References}

Baars, J. W. M., Genzel, R., Pauliny-Toth, I. I. K., \& Witzel, A. 1977, A\&A, 61, 99

Beasley, A. J., Gordon, D., Peck, A. B., et al. 2002, ApJS, 141, 13

Becker, R. H., White, R. L., \& Edwards, A. L. 1991, ApJS, 75, 1 (BWE)

Begelman, M. C. 1996, in Cygnus A - Study of a Radio Galaxy, Proceedings of the Greenbank Workshop, ed. C. L. Carilli, \& D. E. Harris (Cambridge University Press), 209

Bennett, C. L., Lawrence, C. R., Burke, B. F., Hewitt, J. N., \& Mahoney, J. 1986, ApJS, 61, 1 (MITG)

Condon, J. J., Cotton, W. D., Greisen, E. W., et al. 1998, AJ, 115, 1693

Dallacasa, D., Stanghellini, C., Centonza, M., \& Fanti, R. 2000, A\&A, 363, 887

Fanti, R., Fanti, C., Schilizzi, R. T., et al. 1990, A\&A, 231, 333

Fanti, C., Fanti, R., Dallacasa, D., et al. 1995, A\&A, 302, 317

Fanti, C., Pozzi, F., Dallacasa, D., et al. 2001, A\&A, 369, 380

Fomalont, E. B., Petrov, L., MacMillan, D. S., Gordon, D., \& Ma, C. 2003, AJ, 126,2562

Gopal-Krishna, \& Wiita, P. J. 1991, ApJ, 373, 325

Gregory, P. C., \& Condon, J. J. 1991, ApJS, 75, 1011 (87GB)

Gregory, P. C., Scott, W. K., Douglas, K., \& Condon, J. J. 1996, ApJS, 103, 427 (GB6)

Griffith, M., Langston, G., Heflin, M., et al. 1990, ApJS, 74, 129 (MITG)

Griffith, M., Heflin, M., Conner, S., Burke, B., \& Langston, G. 1991, ApJS, 75, 801 (MITG)

Griffith, M. R., Wright, A. E., Burke, B. F., \& Ekers, R. D. 1994, ApJS, 90, 179 (PMNT)

Griffith, M. R., Wright, A. E., Burke, B. F., \& Ekers, R. D. 1995, ApJS, 97, 347 (PMNE)

Kovalev, Y. Y., Kellermann, K. I., Lister, M. L., et al. 2005, AJ, 130, 2473

Kovalev, Y. Y., Petrov, L., Fomalont, E. B., \& Gordon, D. 2007, AJ, 133, 1236

Kraus, A., Krichbaum, T. P., Wegner, R., et al. 2003, A\&A, 401, 161

Labiano, A., Barthel, P. D., O’Dea, C. P., et al. 2007, A\&A, 463, 97

Langston, G. I., Heflin, M. B., Conner, S. R., et al. 1990, ApJS, 72, 621 (MITG)

Marecki, A., Falcke, H., Niezgoda, J., Garrington, S. T., \& Patnaik, A. R. 1999, A\&AS, 135, 273

Mutel, R. L., \& Phillips, R. B. 1988, Proc. 129th IAU Symp., 129, 73

Ochsenbein, F., Bauer, P., \& Marcout, J. 2000, A\&A, 143, 23

O'Dea, C. P. 1998, PASP, 110, 493

O’Dea, C. P., \& Baum, S. A. 1997, AJ, 113, 148

Orienti, M., Dallacasa, D., Tinti, S., \& Stanghellini, C. 2006, A\&A, 450, 959

Ott, M., Witzel, A., Quirrenbach, A., et al. 1994, A\&A, 284, 331

Peck, A. B., \& Taylor, G. B. 2000, ApJ, 534, 90

Petrov, L., Kovalev, Y. Y., Fomalont, E., \& Gordon, D. 2005, AJ, 129, 1163

Petrov, L., Kovalev, Y. Y., Fomalont, E. B., \& Gordon, D. 2006, AJ, 131, 1872

Petrov, L., Kovalev, Y. Y., Fomalont, E. B., \& Gordon, D. 2008, AJ, 136, 580

Readhead, A. C. S., Taylor, G. B., Pearson, T. J., \& Wilkinson, P. N. 1996, ApJ, 460, 634

Snellen, I. A. G., Schilizzi, R. T., de Bruyn, A. G., et al. 1998, A\&AS, 131, 435

Snellen, I. A. G., Schilizzi, R. T., Miley, G. K., et al. 2000a, MNRAS, 319, 445

Snellen, I. A. G., Schilizzi, R. T., \& van Langevelde, H. J. 2000b, MNRAS, 319 , 429

Stanghellini, C., Bondi, M., Dallacasa, D., et al. 1997, A\&A, 318, 376

Stanghellini, C., O'Dea, C. P., Dallacasa, D., et al. 1998, A\&AS, 131, 303

Stanghellini, C., O’Dea, C. P., Dallacasa, D., et al. 2005, A\&A, 443, 891

Torniainen, I., Tornikoski, M., Teräsranta, H., et al. 2005, A\&A, 435, 839

Véron-Cetty, M.-P., \& Véron, P. 2006, A\&A, 455, 773

Vollmer, B., Davoust, E., Dubois, P., et al. 2005a, A\&A, 431, 1177

Vollmer, B., Davoust, E., Dubois, P., et al. 2005b, A\&A, 436, 757

Wenger, M., Ochsenbein, F., Egret, D., et al. 2000, A\&AS, 143, 9

Wright, A. E., Griffith, M. R., Burke, B. F., \& Ekers, R. D. 1994, ApJS, 91, 111 (PMNS)

Wright, A. E., Griffith, M. R., Hunt, A. J., et al. 1996, ApJS, 103, 145 (PMNZ) Xiang, L., Stanghellini, C., Dallacasa, D., \& Haiyan, Z. 2002, A\&A, 385, 768

Xiang, L., Dallacasa, D., Cassaro, P., Jiang, D., \& Reynolds, C. 2005, A\&A, 434, 123

Xiang, L., Reynolds, C., Strom, R. G., \& Dallacasa, D. 2006, A\&A, 454, 729 
B. Vollmer et al.: Quasi-simultaneous observations of inverted-spectrum radio sources, Online Material $p$ l


Fig. 4. continued. 
B. Vollmer et al.: Quasi-simultaneous observations of inverted-spectrum radio sources, Online Material p 2
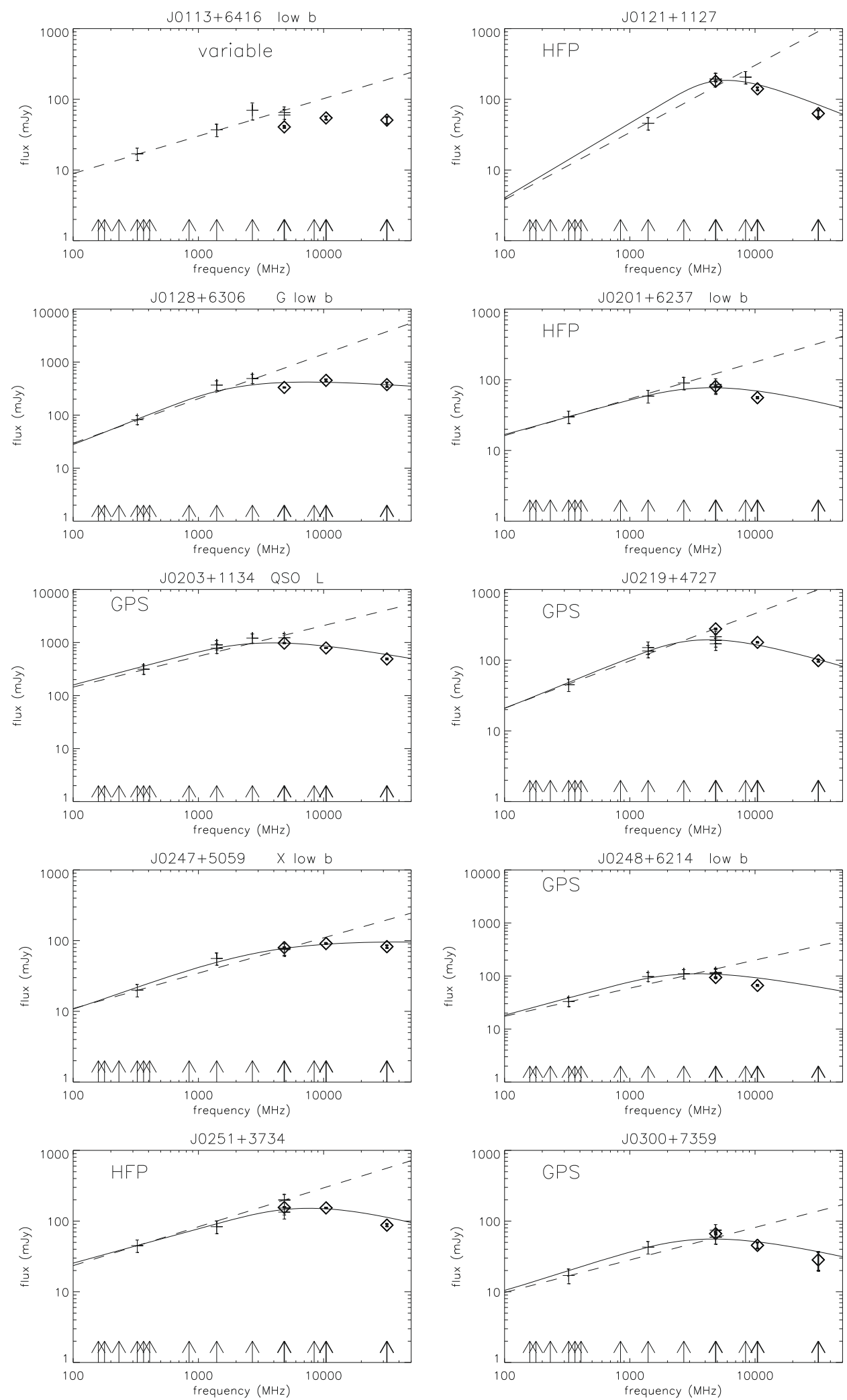

Fig. 4. continued. 
B. Vollmer et al.: Quasi-simultaneous observations of inverted-spectrum radio sources, Online Material p 3
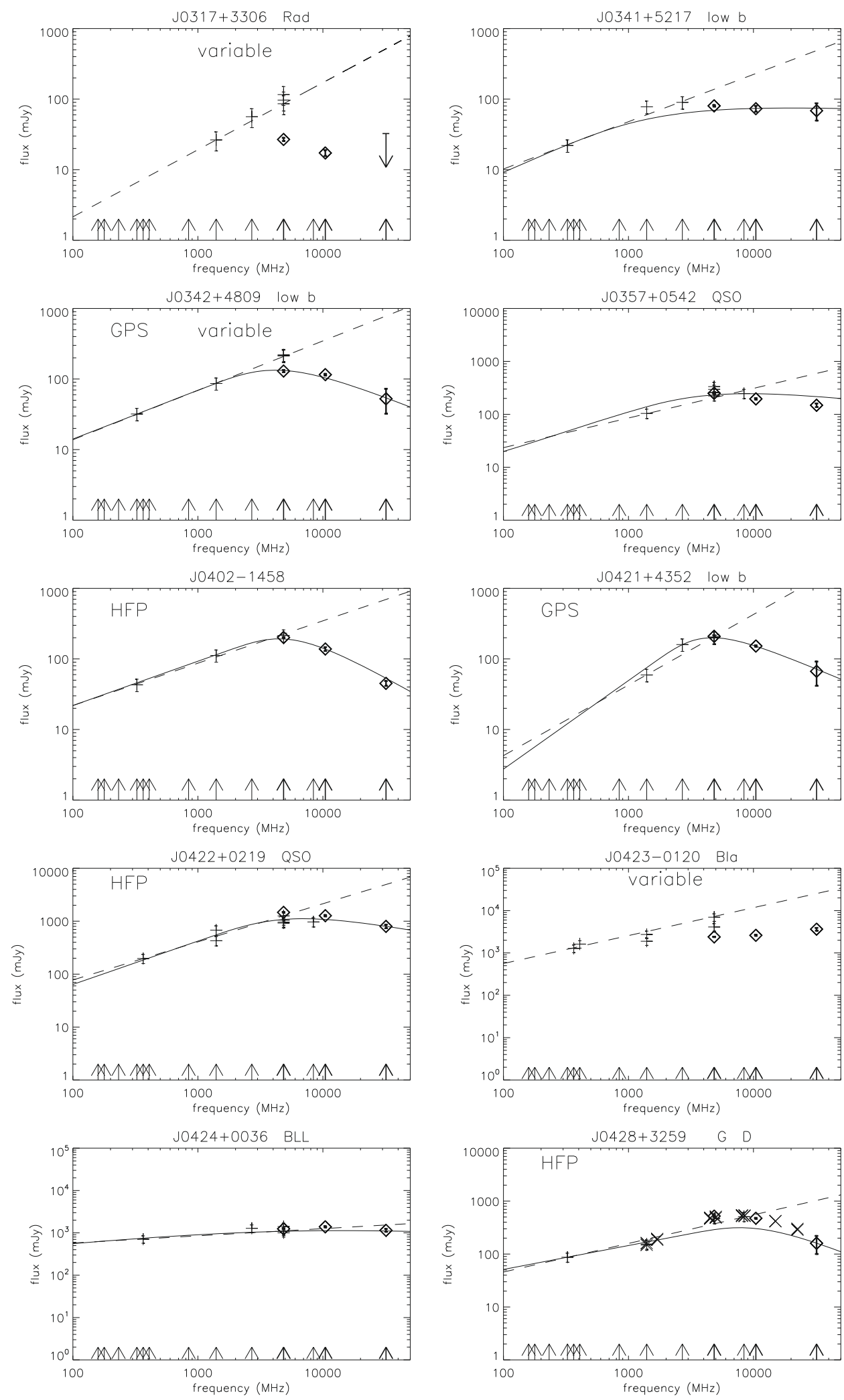

Fig. 4. continued. 
B. Vollmer et al.: Quasi-simultaneous observations of inverted-spectrum radio sources, Online Material p 4
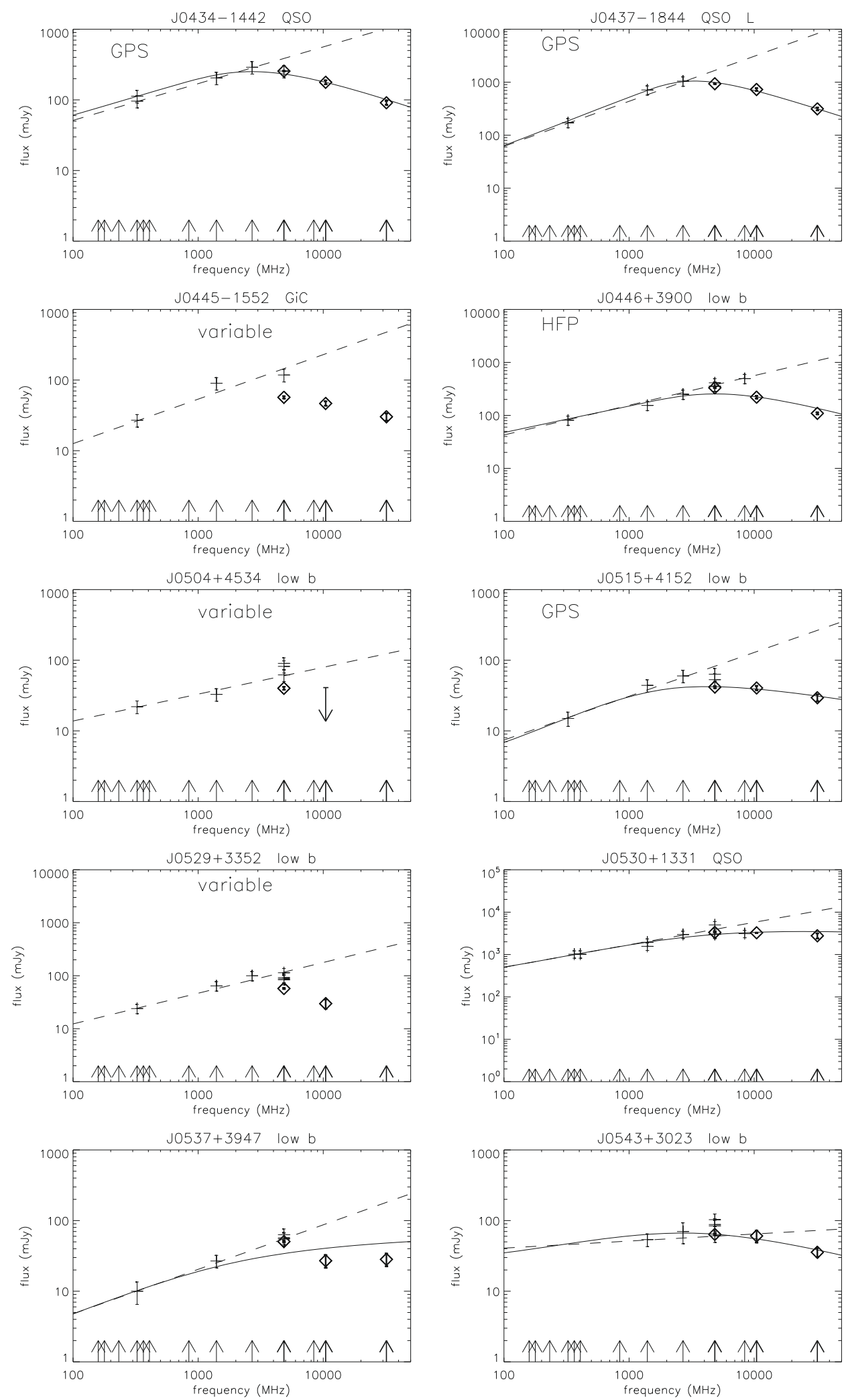

Fig. 4. continued. 
B. Vollmer et al.: Quasi-simultaneous observations of inverted-spectrum radio sources, Online Material p 5
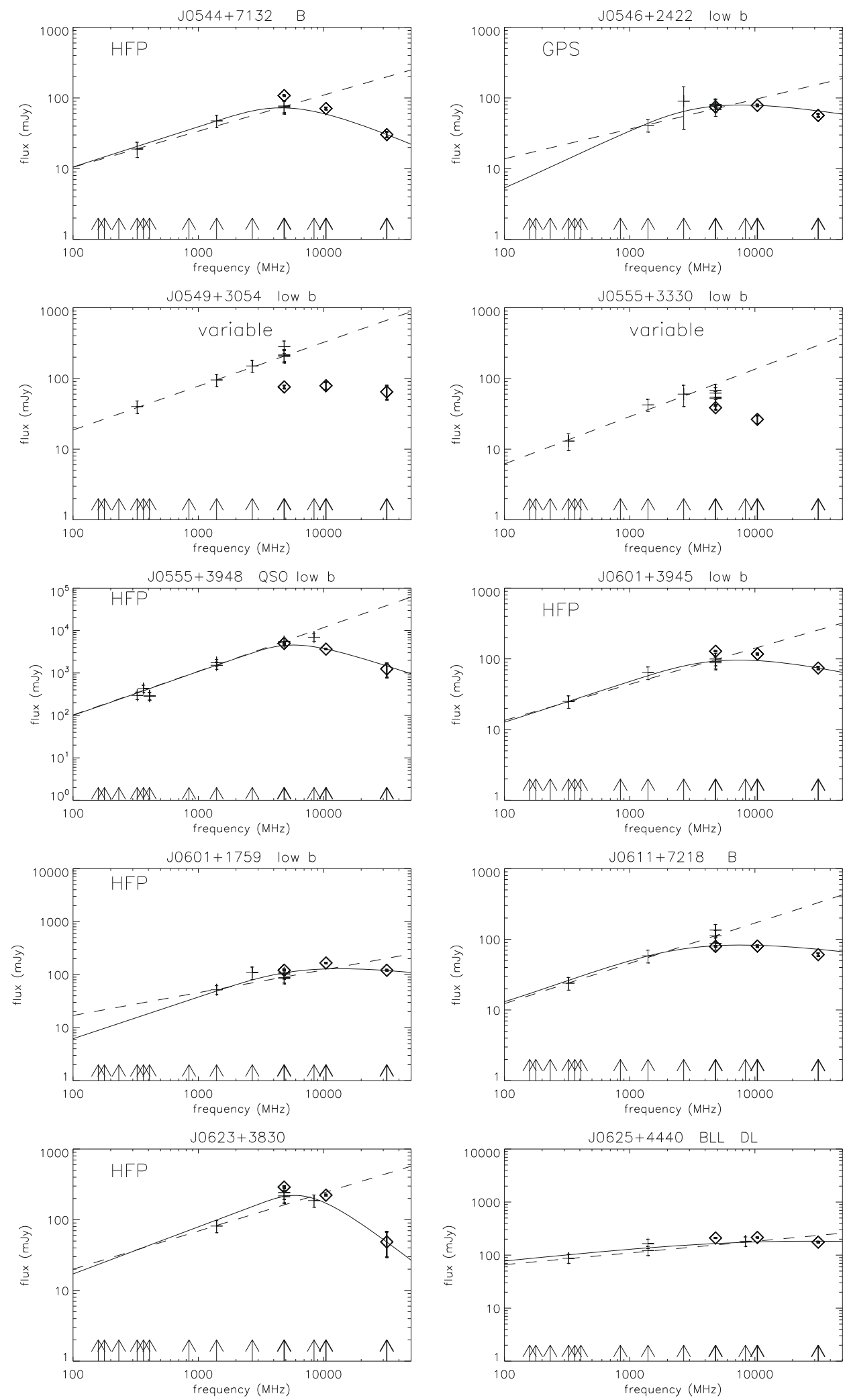

Fig. 4. continued. 
B. Vollmer et al.: Quasi-simultaneous observations of inverted-spectrum radio sources, Online Material p 6
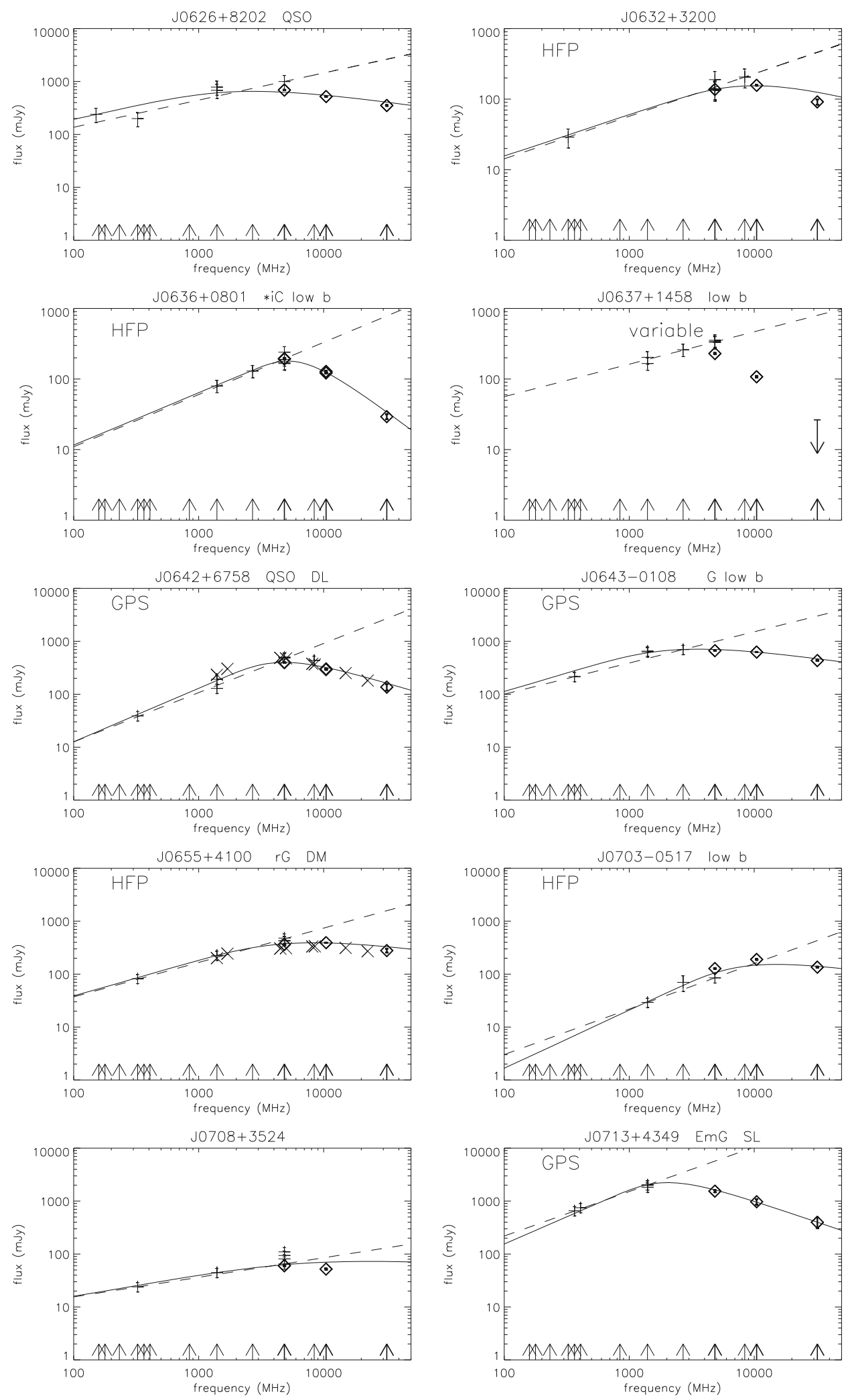

Fig. 4. continued. 
B. Vollmer et al.: Quasi-simultaneous observations of inverted-spectrum radio sources, Online Material $p 7$
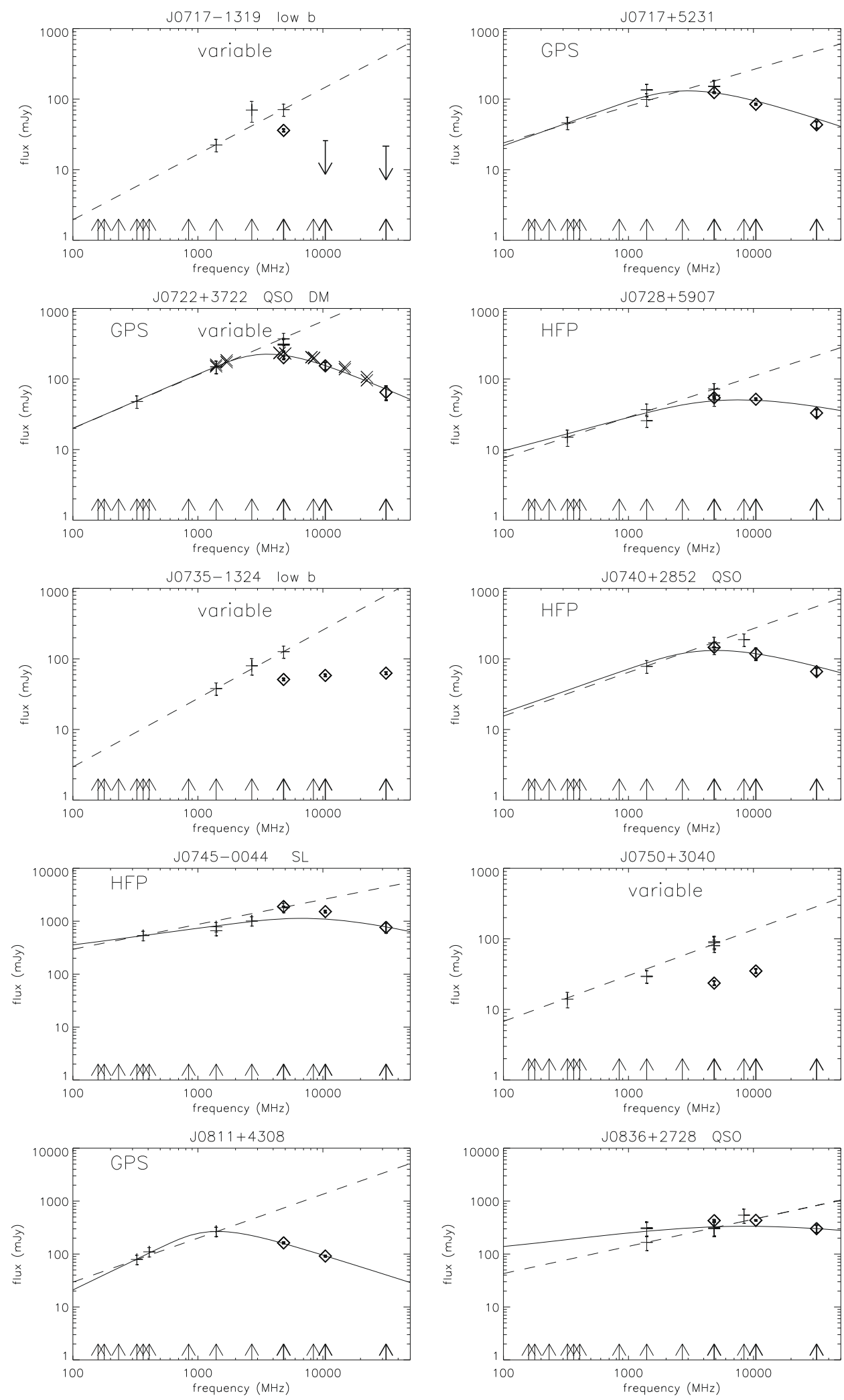

Fig. 4. continued. 
B. Vollmer et al.: Quasi-simultaneous observations of inverted-spectrum radio sources, Online Material p 8


Fig. 4. continued. 
B. Vollmer et al.: Quasi-simultaneous observations of inverted-spectrum radio sources, Online Material p 9
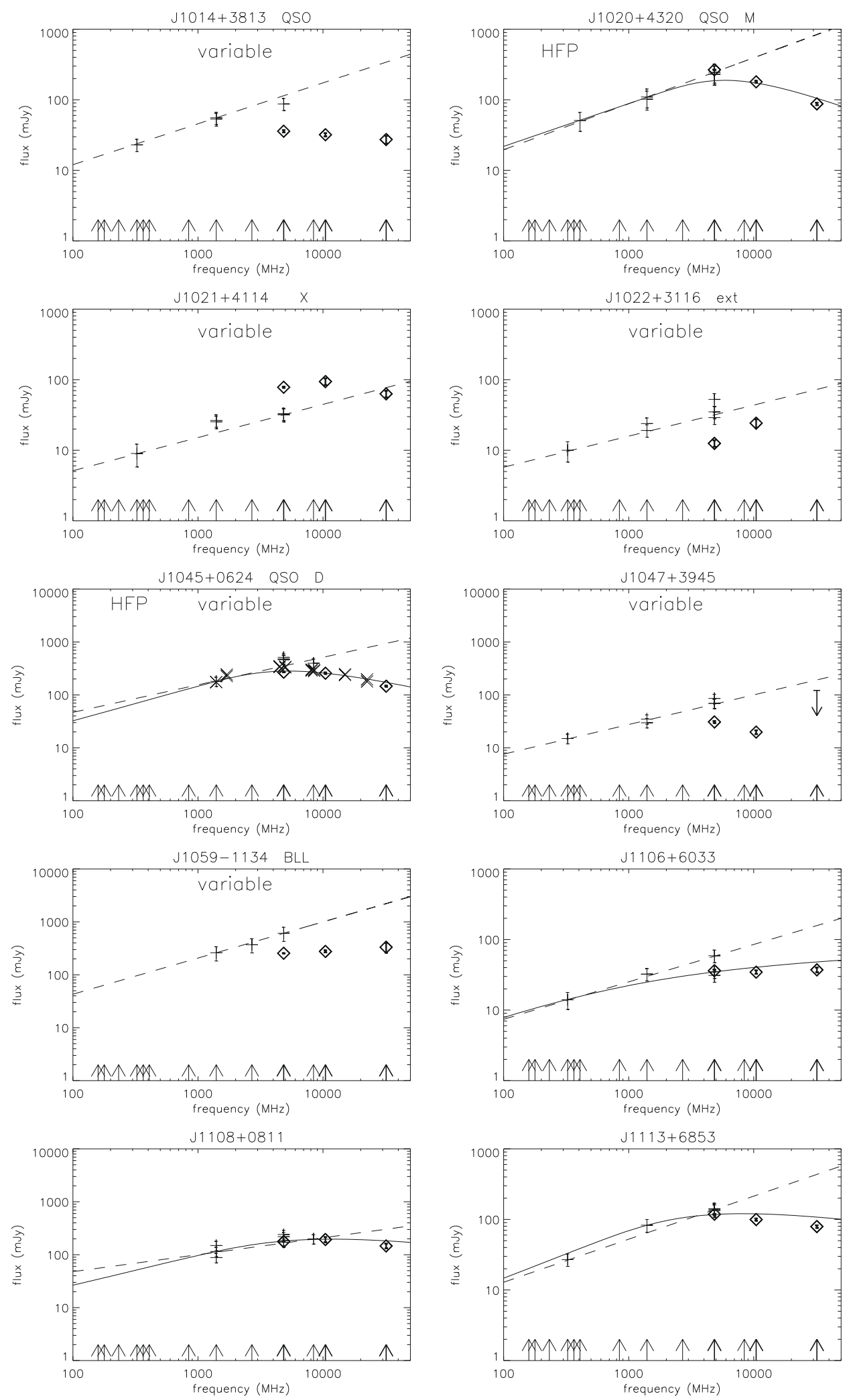

Fig. 4. continued. 
B. Vollmer et al.: Quasi-simultaneous observations of inverted-spectrum radio sources, Online Material p 10
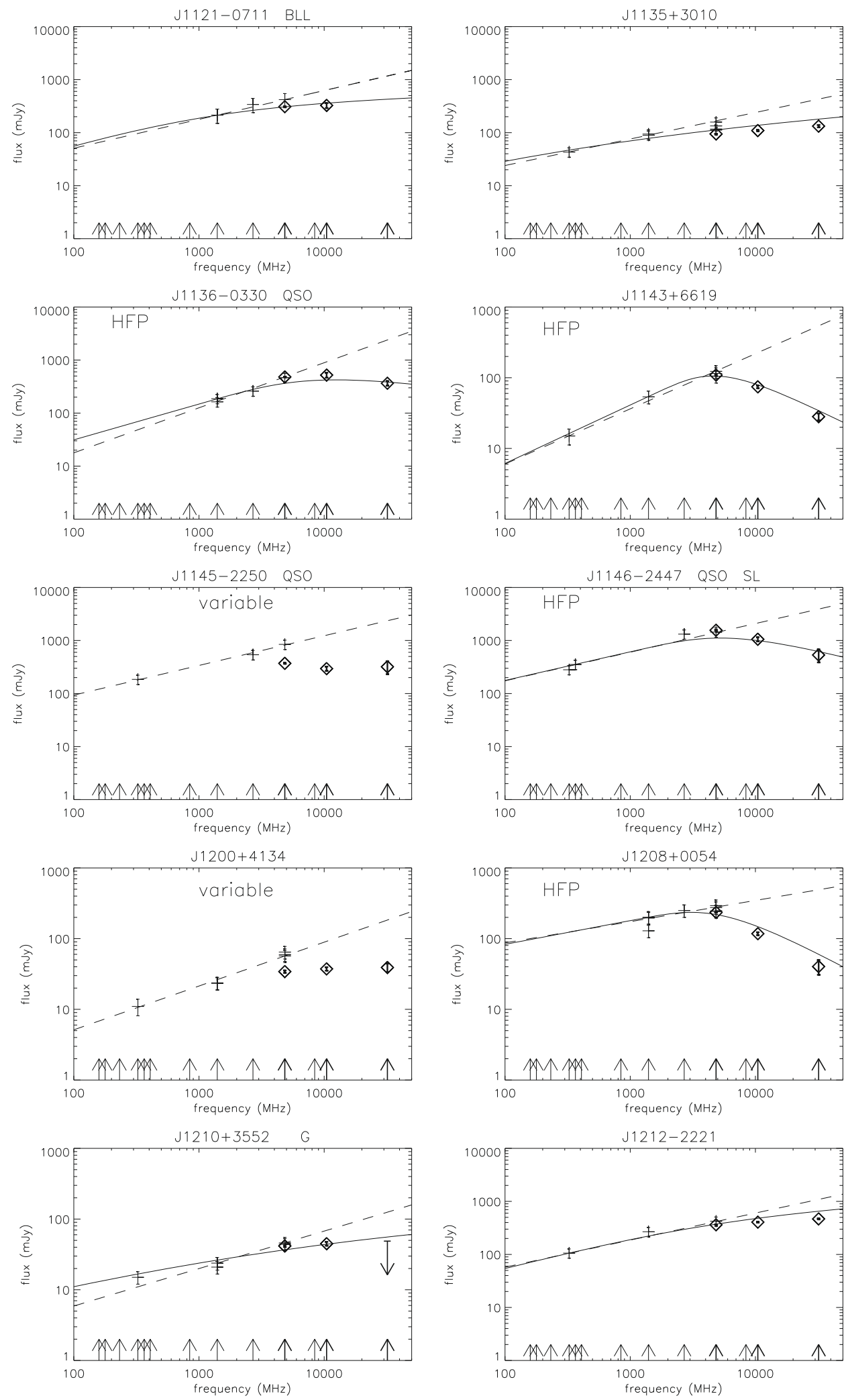

Fig. 4. continued. 
B. Vollmer et al.: Quasi-simultaneous observations of inverted-spectrum radio sources, Online Material p 11
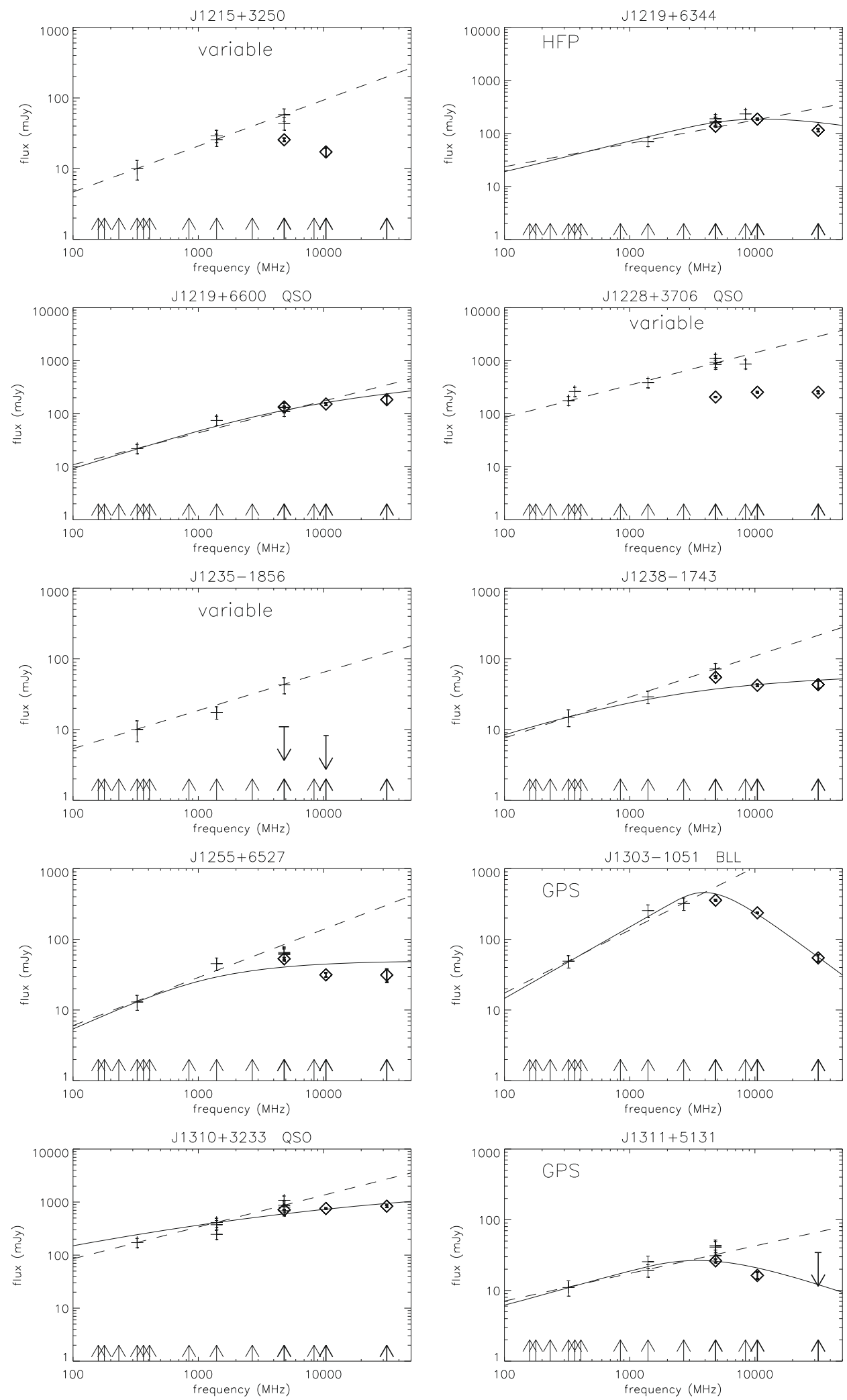

Fig. 4. continued. 
B. Vollmer et al.: Quasi-simultaneous observations of inverted-spectrum radio sources, Online Material p 12
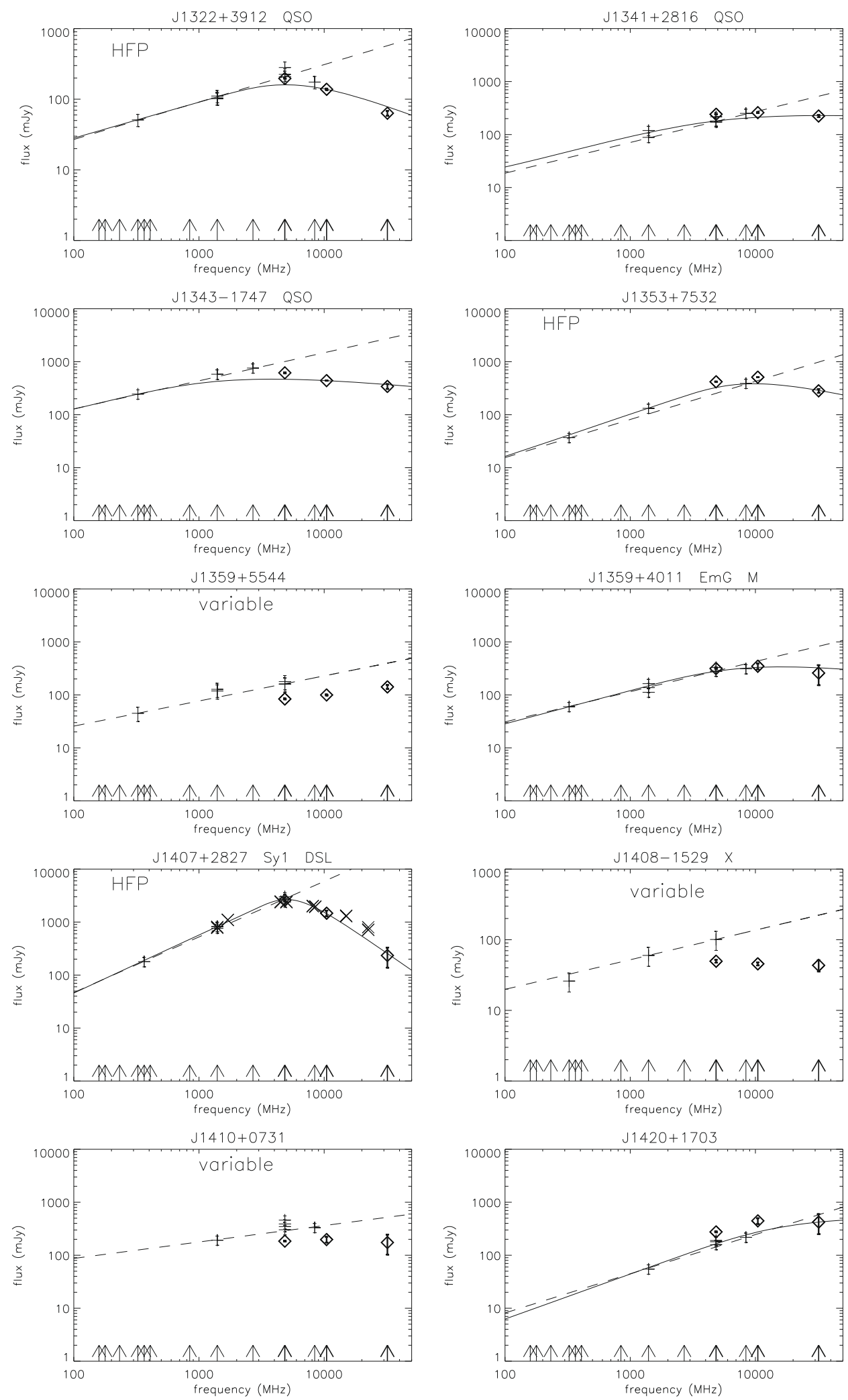

Fig. 4. continued. 
B. Vollmer et al.: Quasi-simultaneous observations of inverted-spectrum radio sources, Online Material p 13
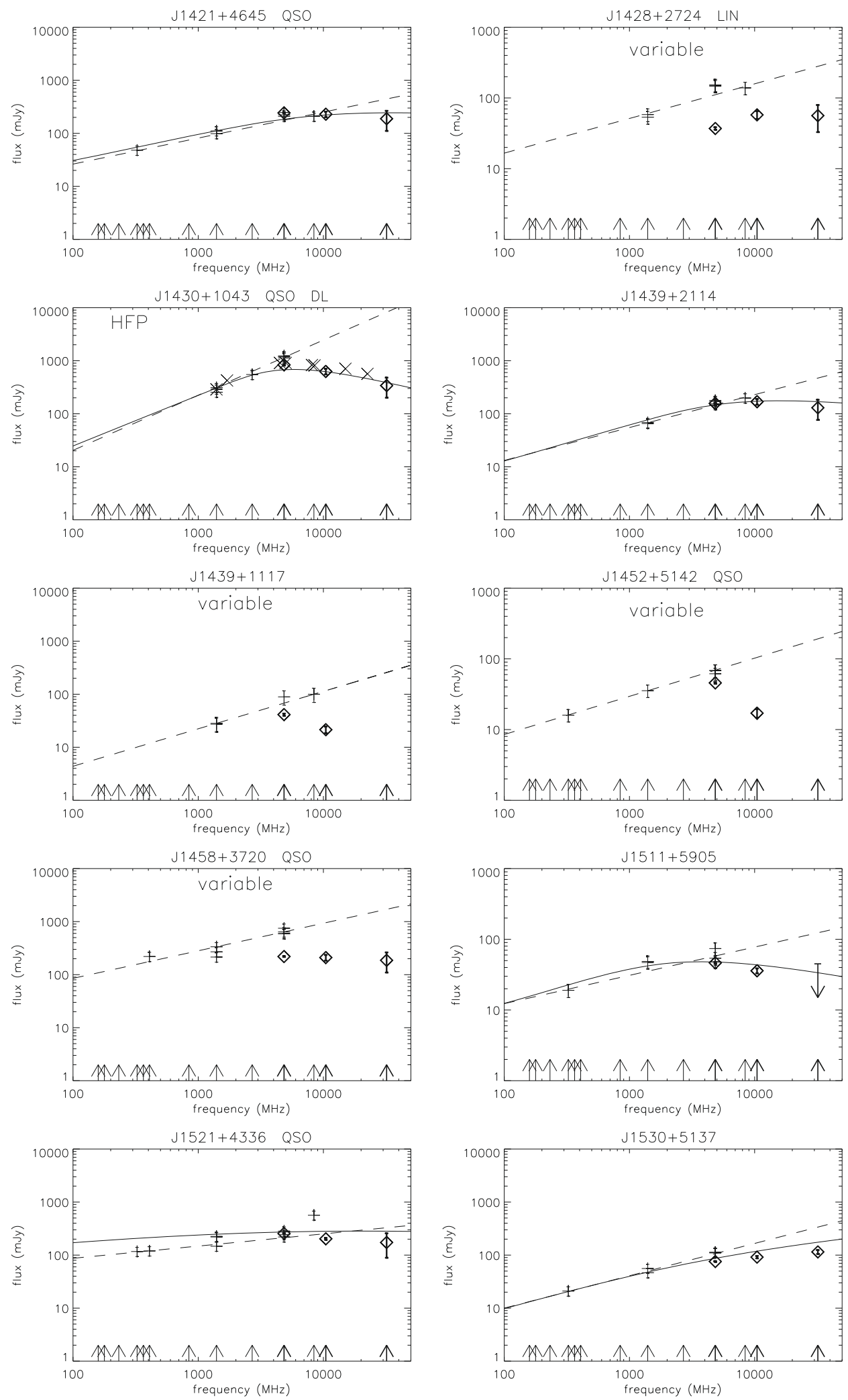

Fig. 4. continued. 
B. Vollmer et al.: Quasi-simultaneous observations of inverted-spectrum radio sources, Online Material p 14
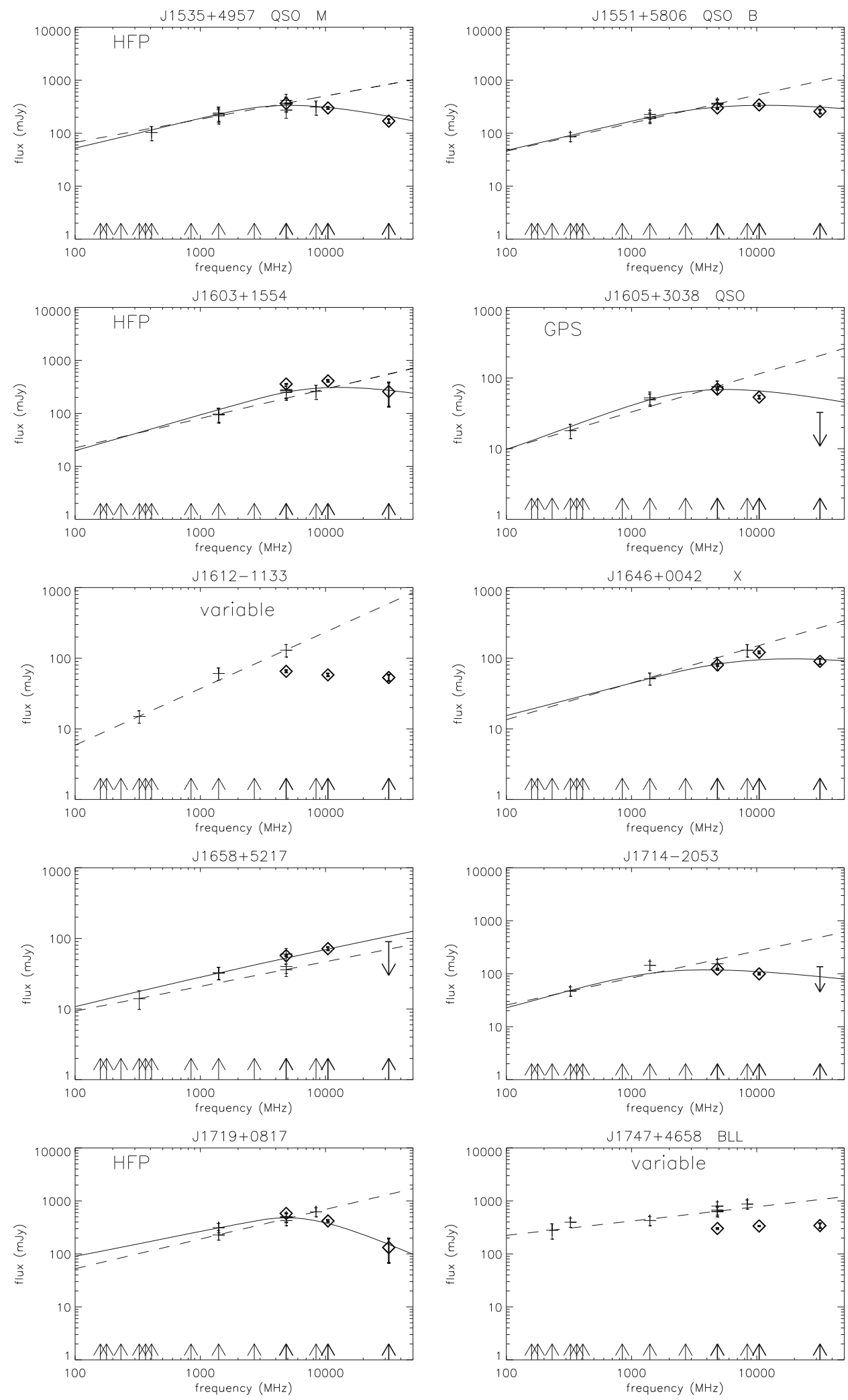

Fig. 4. continued. 
B. Vollmer et al.: Quasi-simultaneous observations of inverted-spectrum radio sources, Online Material p 15
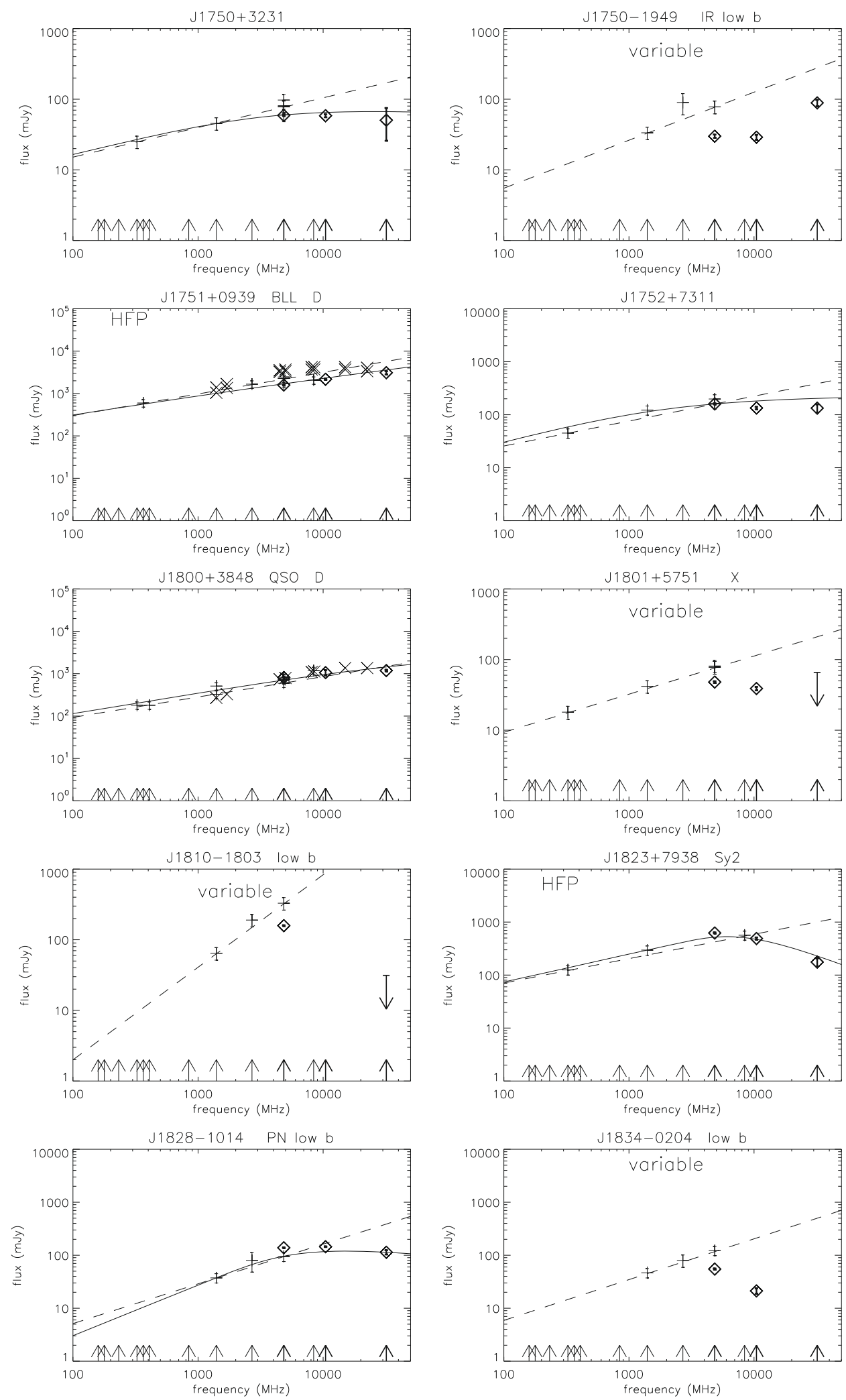

Fig. 4. continued. 
B. Vollmer et al.: Quasi-simultaneous observations of inverted-spectrum radio sources, Online Material p 16
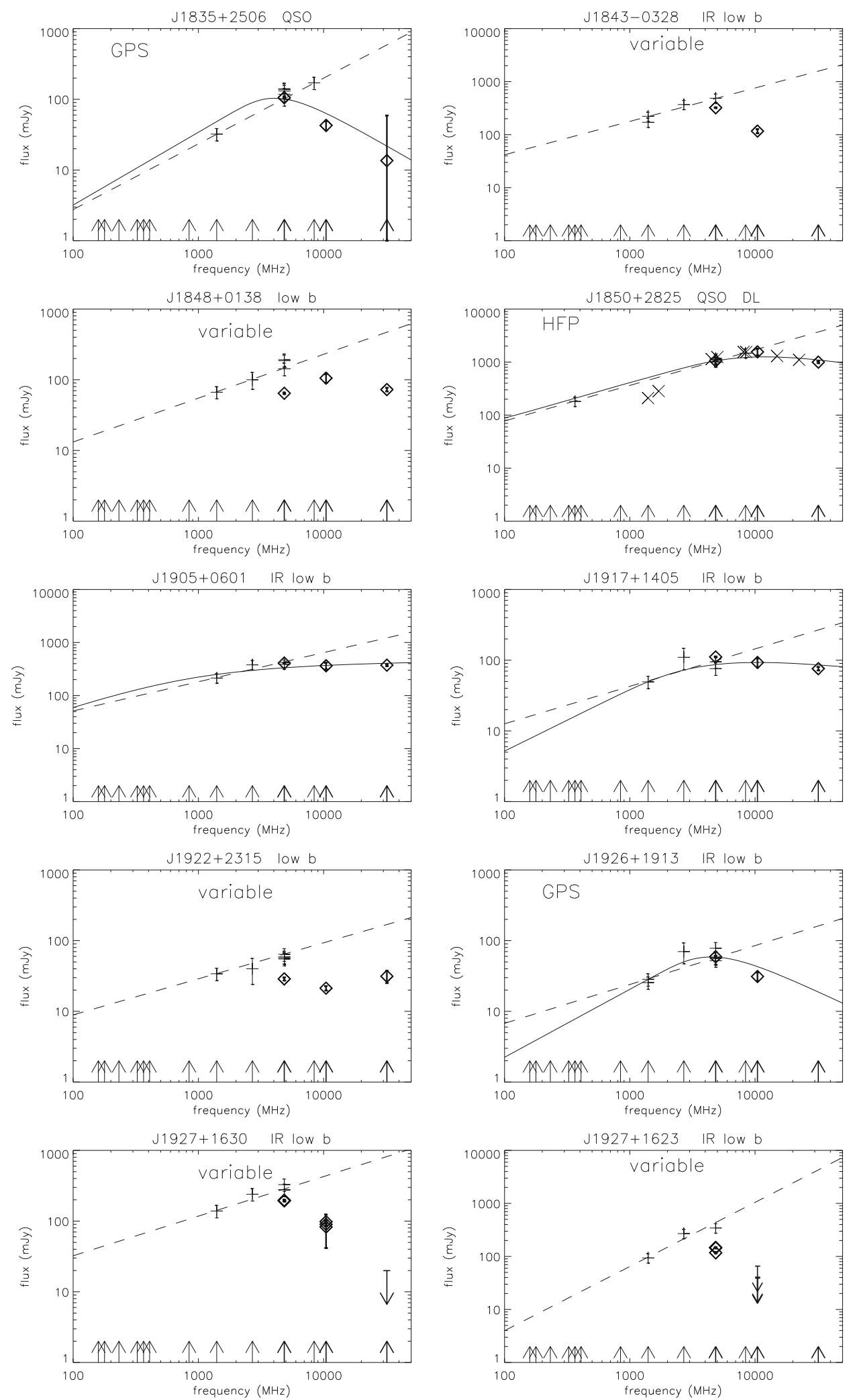

Fig. 4. continued. 
B. Vollmer et al.: Quasi-simultaneous observations of inverted-spectrum radio sources, Online Material p 17
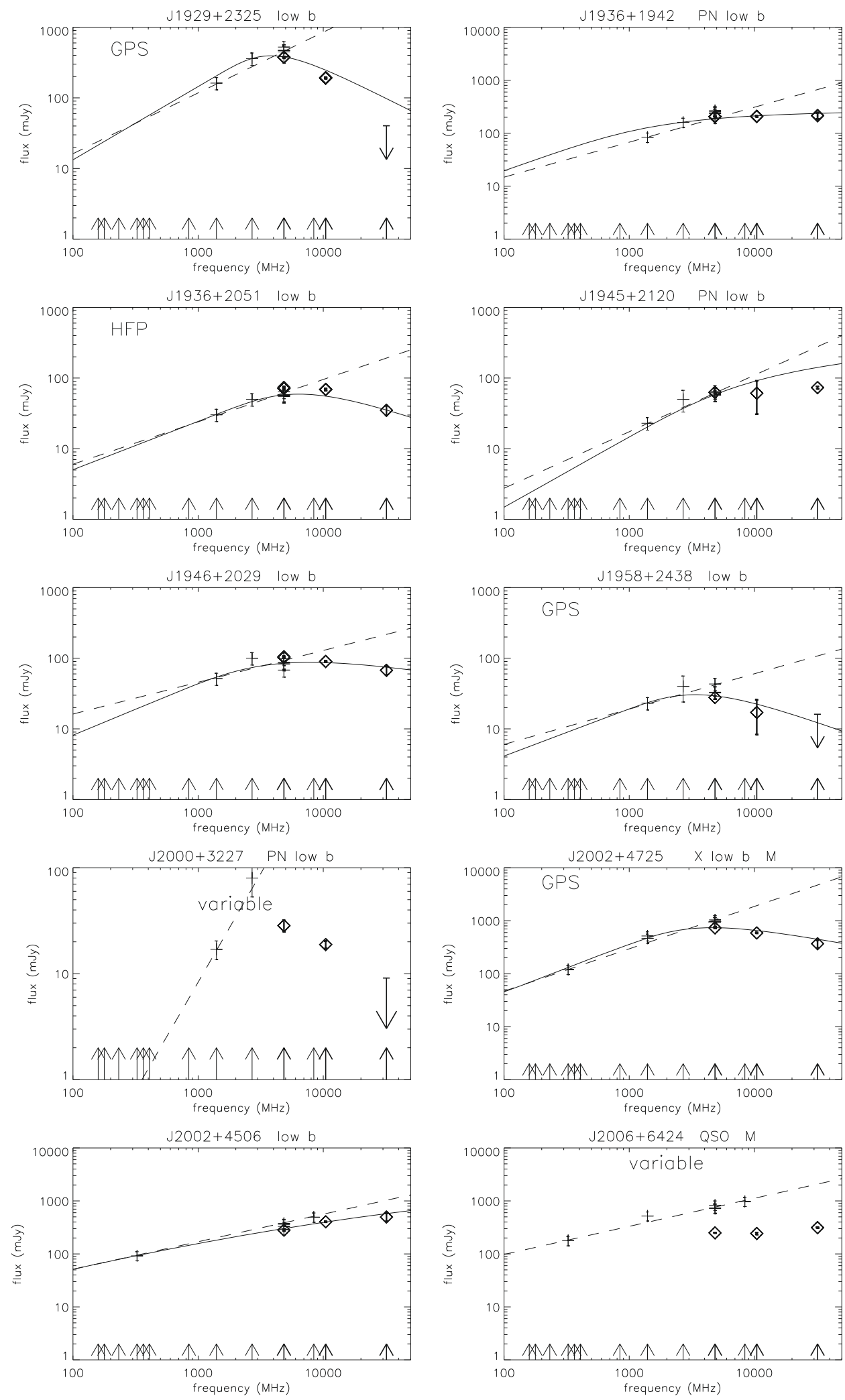

Fig. 4. continued. 
B. Vollmer et al.: Quasi-simultaneous observations of inverted-spectrum radio sources, Online Material p 18
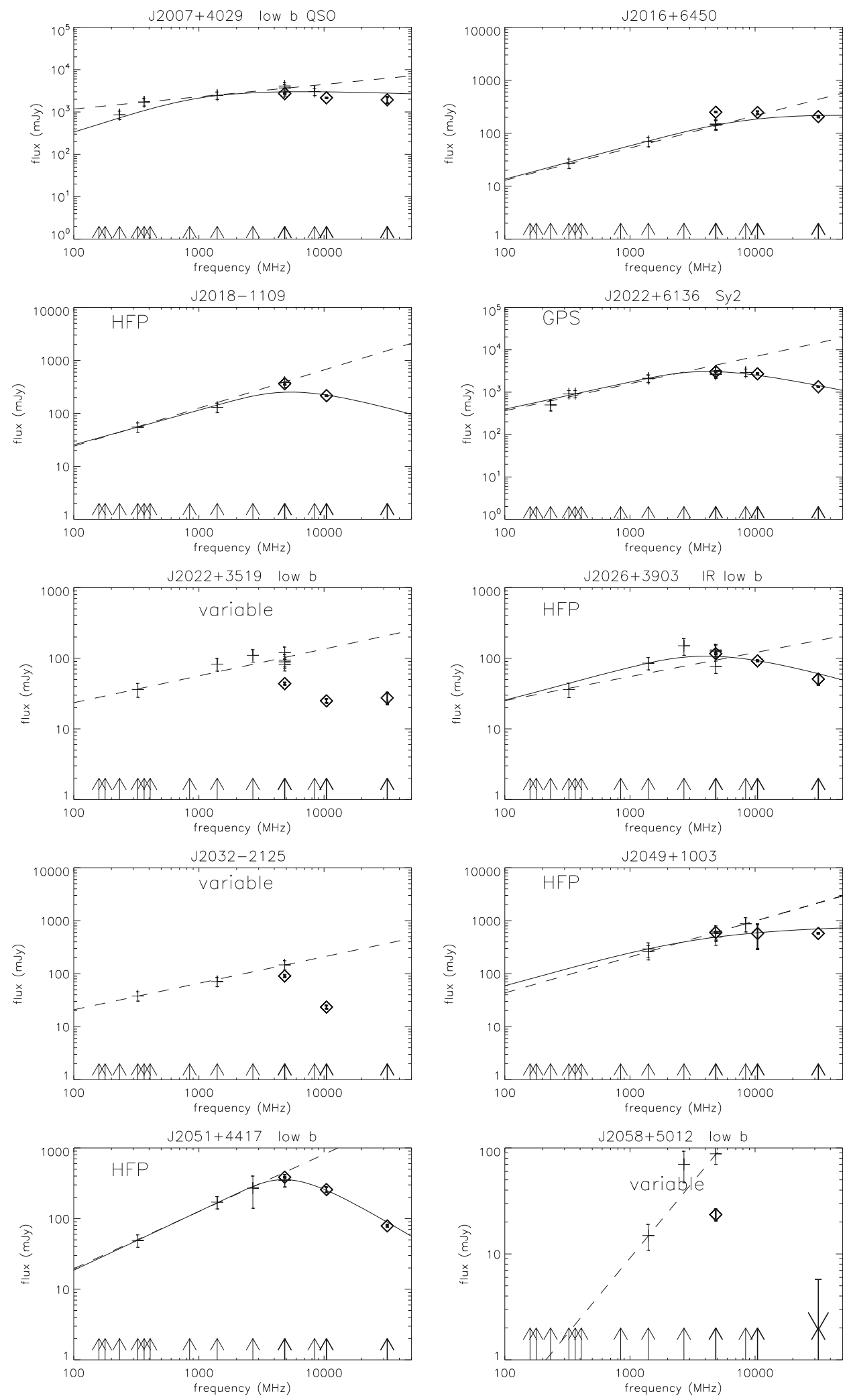

Fig. 4. continued. 
B. Vollmer et al.: Quasi-simultaneous observations of inverted-spectrum radio sources, Online Material p 19
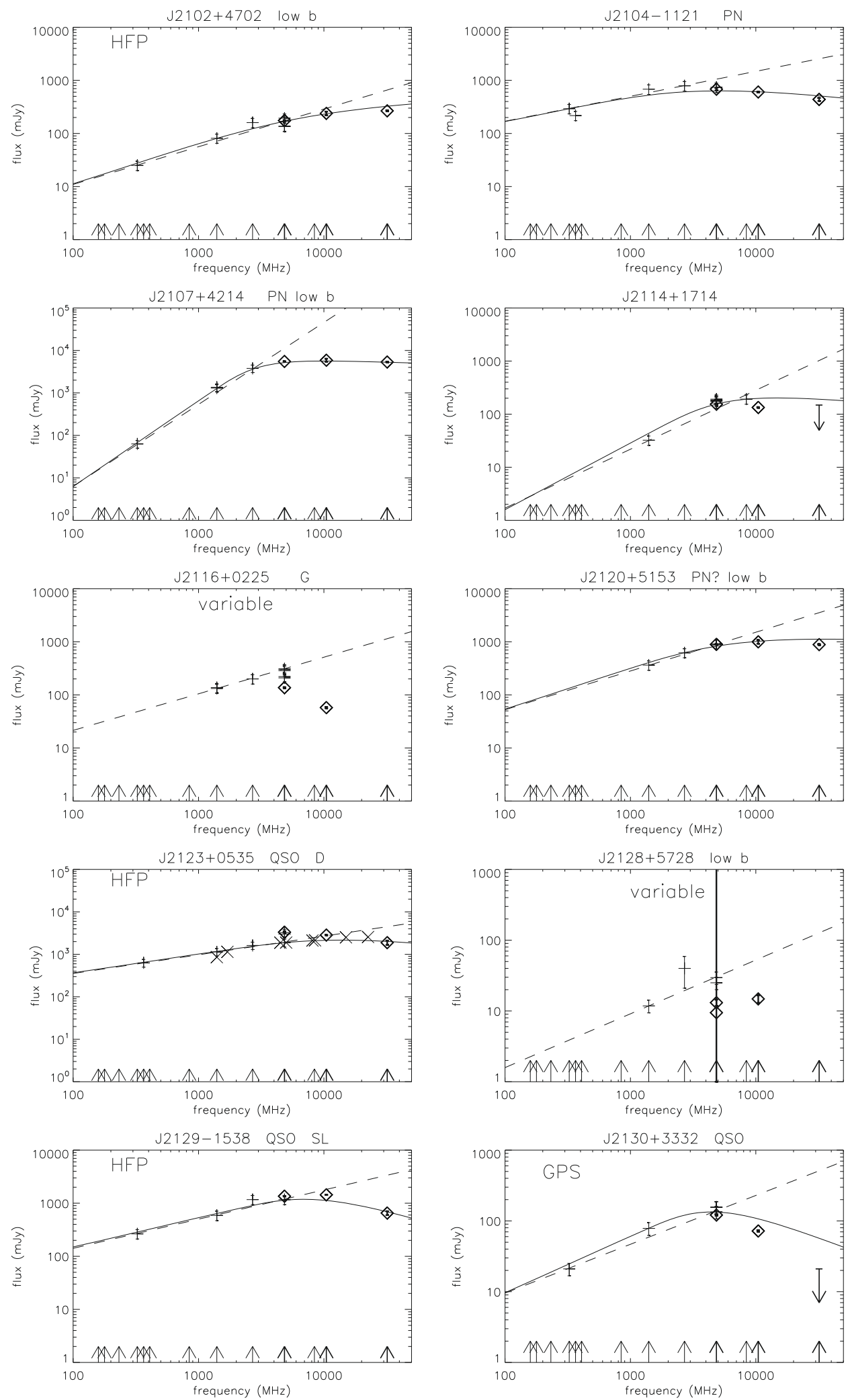

Fig. 4. continued. 
B. Vollmer et al.: Quasi-simultaneous observations of inverted-spectrum radio sources, Online Material p 20
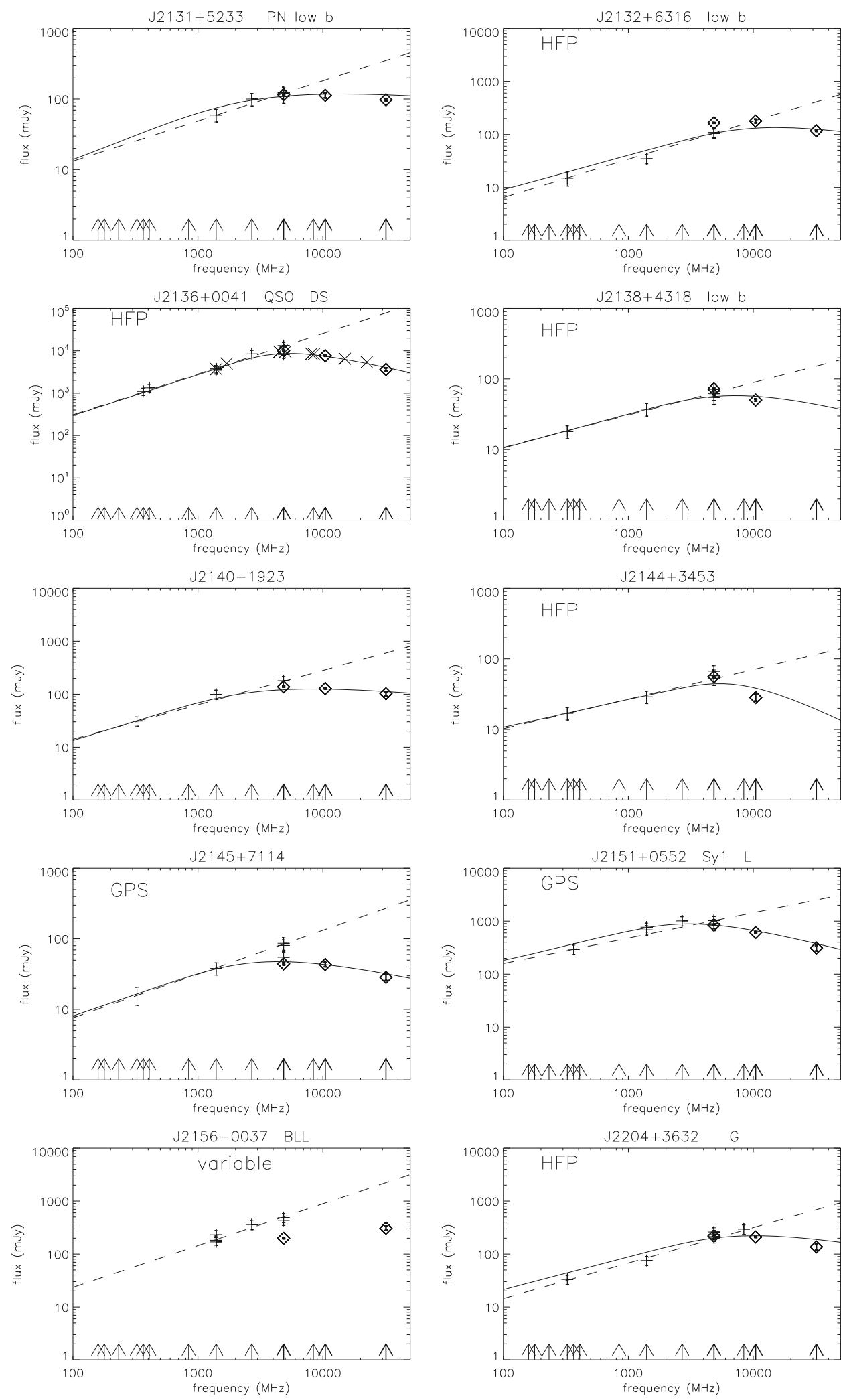

Fig. 4. continued. 
B. Vollmer et al.: Quasi-simultaneous observations of inverted-spectrum radio sources, Online Material p 21


Fig. 4. continued. 
B. Vollmer et al.: Quasi-simultaneous observations of inverted-spectrum radio sources, Online Material p 22
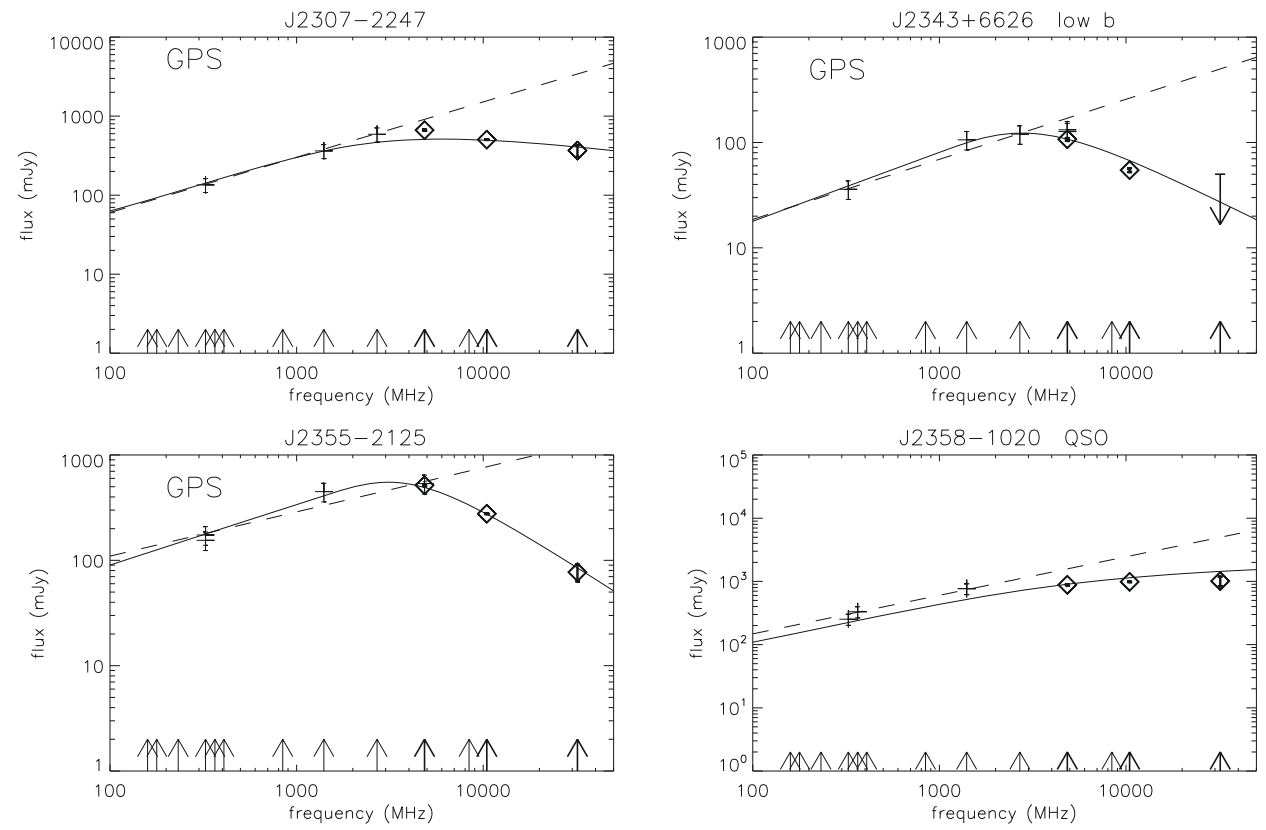

Fig. 4. continued. 